\title{
Gravitational Lensing by Ring-Like Structures
}

\author{
Ethan Lake $^{1 \star}$ and Zheng Zheng ${ }^{1} \dagger$ \\ ${ }^{1}$ Department of Physics and Astronomy, University of Utah,115 South 1400 East, Salt Lake City, UT 84112, USA
}

14 June 2018

\begin{abstract}
We study a class of gravitational lensing systems consisting of an inclined ring/belt, with and without an added point mass at the centre. We show that a common feature of such systems are so-called "pseudo-caustics", across which the magnification of a point source changes discontinuously and yet remains finite. Such a magnification change can be associated with either a change in image multiplicity or a sudden change in the size of a lensed image. The existence of pseudo-caustics and the complex interplay between them and the formal caustics (which correspond to points of infinite magnification) can lead to interesting consequences, such as truncated or open caustics and a non-conservation of total image parity. The origin of the pseudo-caustics is found to be the non-differentiability of the solutions to the lens equation across the ring/belt boundaries, with the pseudo-caustics corresponding to ring/belt boundaries mapped into the source plane. We provide a few illustrative examples to understand the pseudo-caustic features, and in a separate paper consider a specific astronomical application of our results to study microlensing by extrasolar asteroid belts.
\end{abstract}

Key words: gravitational lensing: strong — gravitational lensing: micro — asteroids: general

\section{INTRODUCTION}

Gravitational lensing has developed from an interesting novelty predicted by general relativity (Einstein 1936) to an essential tool in modern astrophysics. It has been applied with great success to (among other things) detect extrasolar planets (e.g. Mao \& Paczynski 1991; Bond et al. 2004; Batista et al. 2014), estimate the masses of galaxy clusters (e.g. Tyson et al. 1998; Bradač et al. 2009; Hoekstra et al. 2013), and determine the large-scale distribution of matter in the Universe (e.g. Wittman et al. 2000; Vikram et al. 2015). In addition, the theoretical study of gravitational lensing systems is of interest in mathematics, namely in catastrophe theory and Morse theory (e.g. Erdl \& Schneider 1993; Petters 1995; Petters et al. 2001).

One common goal of examining lensing systems from a theoretical standpoint is the characterization of the magnification of a source at a given position from the lens mapping. In particular, there exist singularities in the mapping which form caustics (curves or points) in the source plane, where the magnification is formally infinite. Studying the behaviour of these caustics allows for the determination of certain properties of the lens mapping, and provides information about the number of images a source generates. Normally the number of images of a source changes when, and only when, the source crosses a caustic curve, with the total parity of the number of images conserved throughout the source plane. However, some lens models have been found to violate this principle, namely singular isothermal spheres and ellipsoids (e.g. Kovner

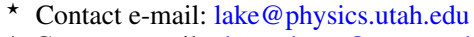

$\uparrow$ Contact e-mail: zhengzheng@astro.utah.edu
}

1987; Evans \& Wilkinson 1998; Rhie 2010) and variants thereof (Wang \& Turner 1997; Shin \& Evans 2008; Tessore \& Metcalf 2015). These systems possess features called "pseudo-caustics", where the number of images of a source can change without the source crossing a caustic.

In this paper, we examine lensing systems consisting of circular rings and belts at various inclinations, with or without a point mass at the centre. We find that these systems possess more general types of pseudo-caustics, characterized by a discontinuous but finite change in the source plane magnification. They are either associated with a change in image multiplicity or with a sudden change in the size of an image. These pseudo-caustics produce complex and interesting magnification patterns, despite the relative simplicity of the lensing systems in question. We aim to obtain a basic understanding of such lensing systems. Although we mainly focus on the limiting case of a sharp belt with a discontinuous mass distribution, we also analyse smooth mass distributions for a better understanding of the origin of the pseudo-caustics and the effect of the smoothing on the pseudo-caustics. We show that the pseudocaustics arise from discontinuities in the lensing mass distribution.

Although our interest here is mainly theoretical and phenomenological, these kind of lensing systems do exist in astronomy, with a relevant example being the asteroid belt around a star. In a related paper (Lake, Zheng, \& Dong 2016), aided by the results presented here, we study the microlensing signatures of extrasolar asteroid belts and explore the possibility of detection with future surveys.

This paper is organized as follows. In Section 2, we briefly recall key gravitational lens equations and present the solutions to the lensing systems we consider. In Section 3, we examine the magni- 
fication patterns and pseudo-caustic structures for each of the systems in consideration and discuss the origin of pseudo-caustics. We summarize our results and discuss implications in Section 4. Several more technical results are presented in a collection of appendices.

\section{SOLUTIONS TO THE LENS EQUATION}

In all the geometries we consider in this paper, a background source is lensed by a mass lying between the source and the observer. We denote the angular position in the image and source plane as $\boldsymbol{r}_{I}=$ $\left(x_{I}, y_{I}\right)$ and $\boldsymbol{r}_{S}=\left(x_{S}, y_{S}\right)$, respectively. We write all angular vectors in units of the Einstein ring radius,

$\theta_{E}=\sqrt{\frac{4 G M}{c^{2}} \frac{D_{L S}}{D_{L} D_{S}}}$,

where $D_{L}, D_{S}$, and $D_{L S}$ are the (angular diameter) distances between the observer and the lens, the observer and the source, and the lens and the source, respectively. The mass $M$ is the total mass of the lens system we consider.

The general lens equation can be written as

$\boldsymbol{r}_{S}=\boldsymbol{r}_{I}-\boldsymbol{\alpha}\left(\boldsymbol{r}_{I}\right)$.

The normalized deflection angle $\alpha$ is found by integrating the contribution over the projected mass distribution of the lens,

$\alpha\left(\boldsymbol{r}_{I}\right)=\frac{1}{\pi} \int \mathrm{d}^{2} \boldsymbol{r}_{I}^{\prime} \kappa\left(\boldsymbol{r}_{I}^{\prime}\right) \frac{\boldsymbol{r}_{I}-\boldsymbol{r}_{I}^{\prime}}{\left|\boldsymbol{r}_{I}-\boldsymbol{r}_{I}^{\prime}\right|^{2}}$,

where $\kappa\left(\boldsymbol{r}_{I}\right)$ is the dimensionless surface mass density of the lens,

$\kappa\left(\boldsymbol{r}_{I}\right)=\frac{\Sigma\left(\boldsymbol{r}_{I}\right)}{\Sigma_{\mathrm{cr}}}$.

The critical surface density $\Sigma_{\mathrm{cr}}$ is defined as

$\Sigma_{\mathrm{cr}} \equiv \frac{c^{2} D_{S}}{4 \pi G D_{L} D_{L S}}$.

For a circularly symmetric lens, $\Sigma_{\text {cr }}$ is equal to the mean surface density inside the Einstein ring radius. The surface density $\kappa$ is connected to the projected gravitational lensing potential $\psi$ (i.e. deflection potential) through Poisson's equation,

$\nabla_{\boldsymbol{r}_{I}}^{2} \psi\left(\boldsymbol{r}_{I}\right)=2 \kappa\left(\boldsymbol{r}_{I}\right)$.

The magnification of the image at the image position $\boldsymbol{r}_{I}$ can be computed as

$\mu\left(\boldsymbol{r}_{I}\right)=\frac{1}{\operatorname{det}(A)}$,

where $\operatorname{det}(A)$ is the determinant of the lensing Jacobian $A_{i j}$, a symmetric matrix formed from the derivatives of the lensing deflection $\boldsymbol{\alpha}=\alpha_{x} \hat{\boldsymbol{x}}_{I}+\alpha_{y} \hat{\boldsymbol{y}}_{I}$,

$A_{i j}=\delta_{i j}-\frac{\partial \alpha_{i}}{\partial x_{j}}$

with $x_{1}=x_{I}$ and $x_{2}=y_{I}$. The total magnification for multiple images is obtained by $\mu_{\text {tot }}=\sum_{i}\left|\mu_{i}\right|$, where $i$ runs over all images. The sign of the magnification reflects the parity of the image. That is, $\mu_{i}>0\left(\mu_{i}<0\right)$ corresponds to positive (negative) parity of the $i$-th image, where the lens mapping is orientation-preserving (orientation-reversing). For derivations of these results, see e.g. Schneider et al. (1992) and Petters et al. (2001).

For the investigation in this paper, we derive the lens equation for a lensing system composed of a thin circular belt with or without a point mass at the centre. We first show the face-on case to build an intuitive understanding and then generalize to inclined belts. For simplicity, we assume the belt to have a uniform surface density. We choose the centre of the belt in projection as the origin of the source and lens planes, and in the case of the inclined belt, we set the directions of the belt's major and minor axes in the lens plane as $\hat{\boldsymbol{x}}_{I}$ and $\hat{\boldsymbol{y}}_{I}$, respectively.

\subsection{Face-on Systems}

As a first step, we consider a uniform thin ring seen face-on, centred at the origin, with radius $a$ (in units of the Einstein ring radius). This lensing geometry has been briefly studied before (Schneider et al. 1992, p.247), and here we present a more detailed analysis.

Rays passing outside the ring $\left(\left|r_{I}\right|>a\right)$ experience a deflection as if the ring were a point mass located at the origin, while rays passing through the ring $\left(\left|r_{I}\right|<a\right)$ are undeflected. The latter result can be easily inferred by considering two chords passing through the impact point with an infinitesimally small opening angle between them - the deflection angles caused by the two arc elements on the ring bounded by the two chords are the same but in the opposite directions, leading to null contribution to the deflection. Thus, the lensing equation reads

$\boldsymbol{r}_{S}=\boldsymbol{r}_{I}-\frac{\boldsymbol{r}_{I}}{\left|\boldsymbol{r}_{I}\right|^{2}} \Theta\left(\left|r_{I}\right|-a\right)$,

where $\Theta(x)$ is the Heaviside step function. Although the lensing equation is written in a vector form, it reduces to a scalar equation (by replacing $\boldsymbol{r}_{S}$ and $\boldsymbol{r}_{I}$ with $r_{S}$ and $r_{I}$, respectively) given the symmetry of the face-on ring/belt case discussed in this subsection. For such a scalar equation, while the source position $r_{S}$ is positive by definition, the image position $r_{I}$ can be either positive or negative, corresponding to images located on the same side $\left(r_{I}>0\right)$ or opposite side $\left(r_{I}<0\right)$ of the lens as the source.

The generalization from a thin ring to a belt of finite width is straightforward. Only the mass inside the circle intersecting the impact point contributes to the deflection, as if it were a point mass at the centre. For a belt of constant surface density with inner and outer radii $a_{i}$ and $a_{o}$, we have

$\boldsymbol{r}_{S}=\boldsymbol{r}_{I}-\frac{\boldsymbol{r}_{I}}{\left|\boldsymbol{r}_{I}\right|^{2}} R\left(\frac{r_{I}^{2}-a_{i}^{2}}{a_{o}^{2}-a_{i}^{2}}\right)$,

where $R(x)$ is a modified ramp function defined as

$R(x)= \begin{cases}0 & \text { if } x \leqslant 0, \\ x & \text { if } 0<x \leqslant 1, \\ 1 & \text { if } x>1 .\end{cases}$

The solution to equation (10) is plotted in Figure 1 for a fixed belt mass with different values of the belt width $\Delta a=a_{o}-a_{i}$. With a mean radius $a_{c}=\left(a_{i}+a_{o}\right) / 2=0.8$, the belt has width ranging from $\Delta a=0.1$ (dark red) to $\Delta a=1.4$ (dark blue). The middle dashed line corresponds to $\boldsymbol{r}_{S}=\boldsymbol{r}_{I}$, which is the solution for the undeflected rays passing interior to the inner edge of the belt $\left(\left|\boldsymbol{r}_{I}\right|<a_{i}\right)$. The left and right dashed curves are the solution for rays deflected by the whole belt (i.e. equivalent to putting all the mass of the belt at the origin), which is the solution for rays passing exterior to the outer edge of the belt $\left(\left|\boldsymbol{r}_{I}\right|>a_{o}\right)$. We refer to the two solutions as the interior and exterior solution, respectively. For rays with $a_{i}<r_{I}<a_{o}$ that pass through the belt, if the belt is wide (low surface density), $\partial r_{S} / \partial r_{I}>0$. However, if the belt is narrow (high surface density), $\partial r_{S} / \partial r_{I}<0$ for $a_{i}<r_{I}<a_{o}$. This is because as 


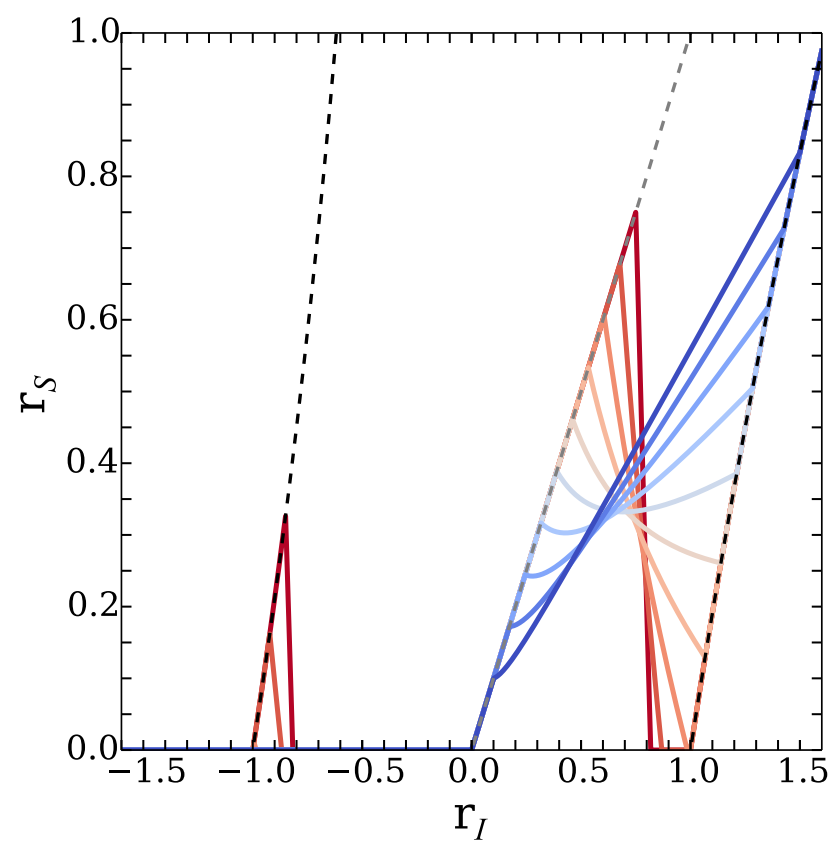

Figure 1. Solutions to the lens equation for a face-on belt with different widths. All belts have equal mass and each has a mean radius of $a_{c}=\left(a_{i}+\right.$ $\left.a_{o}\right) / 2=0.8$ (in units of the Einstein ring radius). The outer (black) dashed curves show the solution to the lensing equation if the belt were replaced by a point mass (with $r_{S}=r_{I}-1 / r_{I}$ ), while the inner (grey) dashed curve shows the solution if the belt had zero mass, and is simply given by $r_{S}=r_{I}$ (i.e. undeflected rays). The solid curves show the physically realized solutions, with the width of the belt increasing from red to blue. Note that in the limit of an infinitesimally thin ring the downward-sloping sections disappear (or become vertical) and the corresponding images vanish, since they become zero-sized. Belts with high enough surface densities (red curves) can form 5 images, with two located on the opposite side of the lens as the source (with $r_{I}<0$ )

$r_{I}$ increases, the high surface density makes a substantial increase in the mass inside the radius of the impact point, which causes a large deflection and allows rays from small $r_{S}$ to be deflected to the observer. The interior and exterior solutions are connected by rays passing through the belt, which complete the final solution. In Figure 1, the number of images formed by a point source at a radius of $r_{S}$ in the source plane is found by counting the number of intersection points of the solution curve with a horizontal line at $r_{S}$.

In the general case, the number of images that can form with a face-on belt lens depends on the configuration of the belt. The lensing equation (10) is linear, quadratic, and quadratic for rays passing interior to the inner edge of the belt, through the belt, and exterior to the outer edge of the belt, respectively. So we can have five solutions in total. Since not all the solutions are physical given the above boundary conditions, we expect the maximum number of images formed by the belt to be $m_{\max }=5$

Let $m\left(\boldsymbol{r}_{S}\right)$ denote the number of images formed by a point source at a location $\boldsymbol{r}_{S}$ in the source plane. We find that in general, wide belts with low surface density have $m\left(\boldsymbol{r}_{S}\right)=m\left(r_{S}\right)=1$ for all $r_{S}$, and $\partial r_{S} / \partial r_{I}>0$ for all $r_{I}$. For narrow belts with high surface densities, we can have multiple images (either $m=3$ or $m=5$ ) for small $r_{S}$, with $\partial r_{S} / \partial r_{I}<0$ for a finite range of $r_{I}$. The transition between these two regimes occurs at the critical belt width

$(\Delta a)_{\mathrm{cr}}=\frac{2}{a_{i}+a_{o}}=\frac{1}{a_{c}}$, which is derived by setting $\partial r_{S} /\left.\partial r_{I}\right|_{r_{I}=a_{i}^{+}}=0$.

In the case of $a_{o}>1$, we find $m\left(r_{S}\right)=1$ for small $r_{S}$ near the origin of the source plane. If $\Delta a>(\Delta a)_{\mathrm{cr}}, m\left(r_{S}\right)=1$ for all $r_{S}$, as $r_{S}$ is a strictly monotonically increasing function of $r_{I}$. If $\Delta a<(\Delta a)_{\mathrm{cr}}$, the belt lens leads to an annulus in the source plane where $m=3$. In the limit of the thin ring, the central image (from rays passing through the belt) disappears, since its size is zero. The remaining two images correspond to undeflected rays passing interior to the ring and rays passing exterior to the ring that are then deflected to the observer.

If $a_{o}<1$, we always have $\Delta a<(\Delta a)_{\mathrm{cr}}$, and as a result $m\left(r_{S}\right)>1$ at some point in the source plane. Intuitively, if $a_{o}<1$, the entire mass of the belt lies within its own Einstein radius (with the belt shrinking to a point mass as the extreme case), and so the belt can form more than one image for small $r_{S}$. We find that $m=5$ near the origin in the source plane and $m=3$ in an annular region surrounding the central $m=5$ region, which becomes an $m=1$ region at large $r_{S}$.

All the above features can be understood precisely by considering the behavior of the total source-plane magnification $\mu\left(r_{S}\right)$ of the lens mapping. Based on the solution of the belt-only case, we see that as a source moves from $r_{S}=0$ to $r_{S} \gg 1, \mu\left(r_{S}\right)$ changes discontinuously as the images (or impact points) cross the belt. The change is caused by the discontinuity in $\partial r_{S} / \partial r_{I}$ at $\left|r_{I}\right|=a_{i}$ or $a_{o}$, which corresponds to a change in the number of images $m$ for a narrow belt with $\Delta a<(\Delta a)_{\text {cr }}$ or a sharp change in the image size for a wide belt with $\Delta a>(\Delta a)_{\mathrm{cr}}$. The loci in the source plane associated with the discontinuous change in $\mu\left(r_{S}\right)$ are defined as pseudo-caustics, which we will define more precisely in $\$ 3$. Since jumps in $\mu\left(r_{S}\right)$ occur when the image of a source crosses the inner or outer edge of the belt, the pseudo-caustics can be found simply by mapping circles with radius $a_{i}$ and $a_{o}$ in the lens (image) plane into the source plane. From this and equation (10), we see that these lensing systems always possess two concentric circular pseudo-caustics, located at source radii

$\mathcal{P S}_{1}=a_{i} \quad$ and $\quad \mathcal{P S}_{2}=\left|a_{o}-\frac{1}{a_{o}}\right|$.

For belts with $\Delta a<(\Delta a)_{\mathrm{cr}}$, which can form regions with $m>1$, the source radii $r_{S}=\mathcal{P} \mathcal{S}_{1}$ and $r_{S}=\mathcal{P S} \mathcal{S}_{2}$ define the boundaries of the different image multiplicity regions mentioned previously.

We now introduce a point mass to our lensing system, located at the centre of the face-on belt. We denote the mass ratio of the belt to the point mass as $q=M_{\text {belt }} / M_{\text {point }}$ and normalize the angular positions by the the Einstein ring radius for the combined belt+point system. This has the effect of adding an extra term (the lensing contribution from the point mass) to the lens equation, with equation (10) becoming

$\boldsymbol{r}_{S}=\boldsymbol{r}_{I}-\frac{1}{1+q} \frac{\boldsymbol{r}_{I}}{\left|\boldsymbol{r}_{I}\right|^{2}}-\frac{q}{1+q} \frac{\boldsymbol{r}_{I}}{\left|\boldsymbol{r}_{I}\right|^{2}} R\left(\frac{r_{I}^{2}-a_{i}^{2}}{a_{o}^{2}-a_{i}^{2}}\right)$.

Just like the belt-only lens, the belt+point mass lens possesses pseudo-caustics which correspond to the inner and outer boundaries of the belt mapped into the source plane. In this case, the pseudo-caustics take the form of two concentric rings at sourceplane radii of

$\mathcal{P S}_{1}=\left|a_{i}-\frac{1}{(1+q) a_{i}}\right| \quad$ and $\quad \mathcal{P S}_{2}=\left|a_{o}-\frac{1}{a_{o}}\right|$.

We can also derive the condition for pseudo-caustics that change the image multiplicity, as opposed to those that just change the size of images. As before, this is done by finding the parameters at 

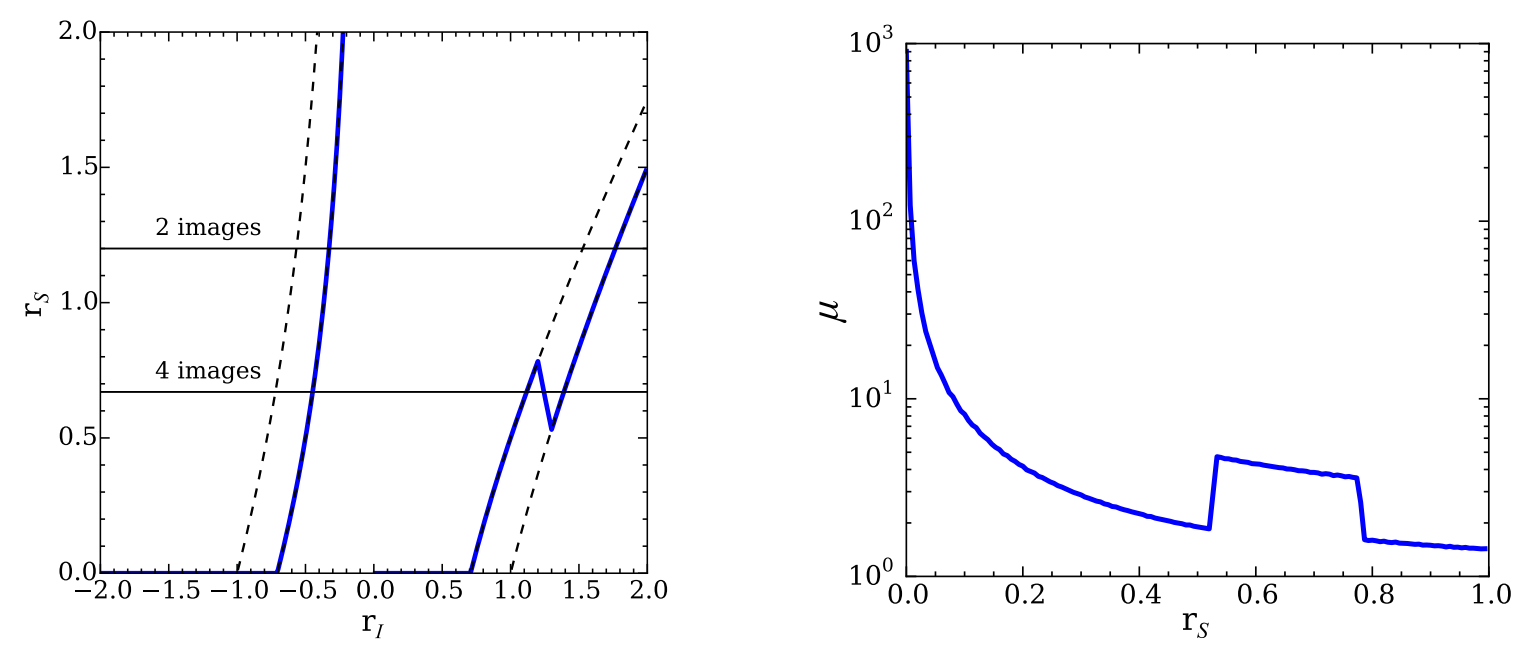

Figure 2. Left: Solutions to the lens equation for a belt+point mass lens. The belt is face-on, with mean radius $a_{c}=1.25$ and width $\Delta a=0.1$. The belt to point mass ratio is set to $q=1.0$. The two inner (outer) black dashed curves correspond to the solutions from just the point mass (from the total belt+point mass system acting as a point mass). The blue solid curves are the physically realized solutions for the belt+point mass case. The presence of the belt creates a region in the source plane where four images can be created. Negative $r_{I}$ corresponds to images located on the opposite side of the lens as the source. Right: Magnification for the lens in the left plot as a function of the distance $r_{S}$ of the (point) source from the origin. The step-like jump in magnification marks the source positions corresponding to the pseudo-caustics (see the text), and the plateau bounded by the two steps corresponds to source positions where four images are formed.

which $r_{S}$ ceases to become a monotonically increasing function of $r_{I}$. Given an inner radius $a_{i}$, we denote $\left(a_{o}\right)_{\mathrm{cr}}$ as the maximum outer radius of the belt that allows for image-forming pseudo-caustics. We obtain

$$
\begin{aligned}
a_{o, \mathrm{cr}}= & \sqrt{\frac{2 q}{1+q+1 / a_{i}^{2}}+a_{i}^{2}}, \quad \text { if } a_{o, \mathrm{cr}}>1 ; \\
a_{o, \mathrm{cr}}= & \frac{1}{\sqrt{2 q+2}}\left[a_{i}^{2}(1+q)+q-1+\left(a_{i}^{4}(1+q)^{2}\right.\right. \\
& \left.\left.+(q-1)^{2}+2 a_{i}^{2} q\left(3 q^{2}+4 q+1\right)\right)^{1 / 2}\right]^{1 / 2}, \quad \text { if } a_{o, \mathrm{cr}} \leqslant 1 .
\end{aligned}
$$

In the belt-only limit $(q \rightarrow+\infty)$, it can be shown that the above equations reduce to equation (12).

The solution to equation (14) is plotted in the left panel of Figure 2 for a face-on belt with $a_{i}=1.2, a_{o}=1.3$, and $q=1$. The two middle curves (with the left one solid blue and the right one blue transitioning to dashed black) correspond to the possible solutions to the lensing equation for rays passing interior to the belt, where only the central point mass is responsible for the deflection. The two dashed curves at large $\left|r_{I}\right|$ are the possible solutions for rays passing exterior to the belt, where the deflection comes from the total mass of the system. The solutions include images located at both positive and negative $r_{I}$, which correspond to images with positive and negative parities, and are located on the same side of the lens as the source and on the opposite side, respectively. The far left dashed curve includes rays deflected from both the belt and the point mass, but the rays in fact pass interior to the belt (i.e. $\left|r_{I}\right|<a_{i}$ ), meaning that this solution is not physically realized. The middle right dashed curve corresponds to rays passing interior to the belt and deflected only by the point mass. For $\left|r_{I}\right|>a_{i}$, deflection from the belt starts to contribute and so this solution is not allowed. Similarly, for the rightmost dashed curve, the allowed solution should have an image position beyond the outer radius of the belt, i.e. $\left|r_{I}\right|>a_{o}$. As a whole, the solid blue curves represent the physically realized solution. The transition from the interior solution to the exterior solution occurs when the images lie within the range of the belt, i.e. when $a_{i}<\left|r_{I}\right|<a_{o}$. Since the belt is narrow and $a_{o}<a_{o, \text { cr }}$, we have $\partial r_{S} / \partial r_{I}<0$ in this part of the solution (see Figure 1 for a comparison).

The right panel of Figure 2 shows the total magnification a point source experiences when lensed by the face-on belt+point system, as a function of distance $r_{S}$ from the origin in the source plane. At both small and large $r_{S}, m=2$ (see the left panel of Figure 2), while in the region bounded by the two pseudo-caustics (see equation (15); which corresponds to $69 / 130 \leqslant r_{S} \leqslant 47 / 60$ for the case here), we find $m=4$. In the magnification map, as the number of images changes from two to four, there is a sharp increase in the magnification (about a factor of 2.5). Note that in this case, the source does not need to cross a formal caustic (defined as a curve in the source plane where $\left|\mu\left(\boldsymbol{r}_{S}\right)\right|=\infty$ for a point source) for the number of images to change. The associated loci across which the magnification is finite but changes discontinuously are the pseudo-causticsmentioned in the introduction. The existence of pseudo-caustics implies that we cannot rely solely on the formal caustics in order to understand these lensing systems.

\subsection{Inclined Systems}

We now allow our system to be inclined, with the normal of the plane of the belt inclined at an angle $i$ relative to the line of sight. We again start with the case of a uniform ring with radius $a$, which becomes an ellipse when seen in projection. The ellipse is parametrized by the set of vectors in the lens (image) plane, which satisfy

$\frac{x^{2}}{a^{2}}+\frac{y^{2}}{b^{2}}=1$, 
where $a$ is the ring's semi-major axis and $b=a \cos i$ is the ring's semi-minor axis. Even for arbitrary inclinations, rays passing interior to the ring are remarkably still undeflected (Chandrasekhar 1969; Bray 1984).

To find the deflection for an impact point $\boldsymbol{r}_{I}$ in the image plane that lies outside the ring (i.e. the exterior solution), we consider an ellipse passing through $\boldsymbol{r}_{I}$ and confocal to the projected ring. The confocal ellipse is expressed as

$\frac{x^{2}}{a^{\prime 2}}+\frac{y^{2}}{b^{\prime 2}}=1$,

where the primed semi-axes are defined by $a^{\prime 2}=a^{2}+\lambda$ and $b^{\prime 2}=$ $b^{2}+\lambda$, with $\lambda$ found by substituting $\boldsymbol{r}_{I}=\left(x_{I}, y_{I}\right)$ into equation (18) and taking the positive solution.

The deflection angle $\boldsymbol{\alpha}_{r}\left(\boldsymbol{r}_{I}\right)=\alpha_{1} \hat{\boldsymbol{x}}_{I}+\alpha_{2} \hat{\boldsymbol{y}}_{I}$ from the ring can be found analytically using results from ellipsoidal potential theory (Bourassa \& Kantowski 1975), which are solved nicely in Schramm (1990). For $\boldsymbol{r}_{I}=x_{I} \hat{\boldsymbol{x}}_{I}+y_{I} \hat{\boldsymbol{y}}_{I}$, the deflection angle from the ring is

$\boldsymbol{\alpha}_{r}\left(\boldsymbol{r}_{I} ; a, i\right)=p^{\prime 2}\left(\frac{\boldsymbol{x}_{I}}{a^{\prime 3} b^{\prime}}+\frac{\boldsymbol{y}_{I}}{a^{\prime} b^{\prime 3}}\right)$,

where $p^{\prime}=\left(x_{I}^{2} / a^{4}+y_{I}^{2} / b^{\prime 4}\right)^{-1 / 2}$ is the perpendicular distance from the centre of the confocal ellipse to the tangent line at $\boldsymbol{r}_{I}$ and we also use the notation $\boldsymbol{x}_{I}=x_{I} \hat{\boldsymbol{x}}_{I}$ and $\boldsymbol{y}_{I}=y_{I} \hat{\boldsymbol{y}}_{I}$ for simplicity.

To obtain an expression for the deflection caused by a belt, we make use of more results in ellipsoidal potential theory. Consider a circular uniform disc in projection, which appears as an elliptical disc with constant normalized surface density $\kappa_{0}$, semi-major axis $a$, and inclination $i$. The normalized deflection angle at a point $\boldsymbol{r}_{\boldsymbol{I}}$ in the image plane is (Schramm 1990)

$\alpha_{d}\left(\boldsymbol{r}_{I} ; a, i\right)=2 \pi \tilde{a} \tilde{b} \kappa_{0}\left(\frac{1}{a^{\prime}+b^{\prime}} \frac{\boldsymbol{x}_{I}}{a^{\prime}}+\frac{1}{a^{\prime}+b^{\prime}} \frac{\boldsymbol{y}_{I}}{b^{\prime}}\right)$,

where for points interior to the disc, we set $a^{2}=\tilde{a}^{2}=x_{I}^{2}+y_{I}^{2} / \cos ^{2} i$ and $b^{\prime}=\tilde{b}=a^{\prime} \cos i$. For points exterior to the disk, we set $\tilde{a}=a$ and $\tilde{b}=b$ and obtain $a^{\prime}$ and $b^{\prime}$ by substituting $\left(x_{I}, y_{I}\right)$ into equation (18).

To calculate the deflection angle of a belt of inner and outer semi-major axes $a_{i}$ and $a_{o}$, we superimpose a disc with semi-major axis $a_{o}$, inclination $i$, and surface density $\kappa_{0}$ with a disc of effective surface density $-\kappa_{0}$, semi-major axis $a_{i}$, and inclination $i$. The total deflection from the two superimposed discs is just that from a uniform belt with inner and outer radii $a_{i}$ and $a_{o}$ :

$\boldsymbol{\alpha}_{b}\left(\boldsymbol{r}_{I} ; a_{i}, a_{o}, i\right)=\boldsymbol{\alpha}_{d}\left(\boldsymbol{r}_{I} ; a_{o}, i\right)-\boldsymbol{\alpha}_{d}\left(\boldsymbol{r}_{I} ; a_{i}, i\right)$.

Here, $\kappa_{0}$ is defined as $\kappa_{0}=1 /\left[\pi\left(a_{o} b_{o}-a_{i} b_{i}\right)\right]$ for the belt so that the total dimensionless mass of the belt is unity. It is easy to verify that the belt does not deflect rays that pass within the inner edge of the belt.

With the deflection angle from the belt, the lens equation for the general case of an inclined belt+point system then becomes

$\boldsymbol{r}_{S}=\boldsymbol{r}_{I}-\frac{1}{1+q} \frac{\boldsymbol{r}_{I}}{\left|\boldsymbol{r}_{I}\right|^{2}}-\frac{q}{1+q} \boldsymbol{\alpha}_{b}\left(\boldsymbol{r}_{I} ; a_{i}, a_{o}, i\right)$,

where $q$ is the belt-to-point mass ratio. The second term on the right-hand side corresponds to the deflection from the centre point mass. Taking the limit of $q \rightarrow+\infty$ recovers the equation for the belt-only case.

While computing the deflection for systems with arbitrary inclination is straightforward, analytically solving the lensing equation becomes much more cumbersome. However, it is possible to get a relatively simple analytic classification for the two limiting cases of the inclination $i$ - we have addressed the face-on $i=0$ case in the previous section, and present an analysis of the edge-on $i=\pi / 2$ case in Appendix A.

Equipped with the solutions of the deflection angle and the lens equation for the inclined system and using our understanding of the face-on system, we turn now to an investigation of the general lensing properties of belt+point mass systems as a function of the inclination.

\section{LENSING PROPERTIES OF BELT+POINT MASS SYSTEMS: CAUSTICS AND PSEUDO-CAUSTICS}

To study the lensing properties of belt+point lensing systems, we employ the inverse ray-shooting technique (e.g. Schneider \& Weiss 1986). Light rays are drawn uniformly from the image plane and mapped back to the source plane according to the lens equation. The magnification at each position in the source plane is computed as the ratio of the density of rays at this angular position to that in the image plane, which is also the density of rays in the source plane in the absence of the lens.

Of interest in analysing the properties of lensing systems is the behaviour of caustic curves, which are loci in the source plane where the Jacobian matrix of the lens mapping is singular $(\operatorname{det}(A)=0)$, and where the magnification $\mu\left(\boldsymbol{r}_{S}\right)$ is formally infinite. The caustic curves correspond to the singular points of the lens mapping. For smooth lens mappings, we may invoke Sard's theorem to show that the set of caustic curves must have measure zero, which means that they must consist of collections of onedimensional curves and isolated points.

For all but the most simple lensing geometries, finding analytical expressions for the caustic curves is difficult, since the mapping $\boldsymbol{r}_{I} \mapsto \boldsymbol{r}_{S}$ is highly non-linear. While the inverse-ray shooting technique enables us to find high magnification regions, it is limited by the resolution of the mapping. To better locate the caustic curves, we use equation (7) to track down points where the magnification is above some threshold value (we adopt $\left|\mu\left(\boldsymbol{r}_{S}\right)\right|>1000$ ). In Appendix $\mathrm{B}$, we provide analytic expressions for the magnification in each of the systems we consider.

For isolated lensing systems ${ }^{1}$ with lensing potentials $\psi\left(\boldsymbol{r}_{I}\right)$ that are smooth for all $\boldsymbol{r}_{I}$ away from a finite number of point singularities, several results from singularity theory can be applied to constrain the types of images that sources can form. In particular, the image multiplicity $m\left(\boldsymbol{r}_{S}\right)$ can only ever change by two. This can be seen by applying the Poincare-Hopf index theorem, which states that the sum $\sum_{i} \operatorname{sgn}\left(\mu_{i}\right)$ is a constant for all non-caustic source locations $\boldsymbol{r}_{S}$, and implies that images can only be created / annihilated in opposite-parity pairs (Burke 1981; McKenzie 1985). For mass distributions $\kappa\left(\boldsymbol{r}_{I}\right)$ with no point singularities, this implies that the image multiplicity $m\left(\boldsymbol{r}_{S}\right)$ must be odd for all $\boldsymbol{r}_{S}$, since we expect a source at $\boldsymbol{r}_{S}$ to form only one, undeflected image with positive parity in the limit $\left|\boldsymbol{r}_{S}\right| \rightarrow \infty$. More generally, if $\psi\left(\boldsymbol{r}_{I}\right)$ possesses $g$ point singularities, the total image multiplicity is always odd (even) if $g$ is even (odd), with a source at $\left|\boldsymbol{r}_{S}\right| \rightarrow \infty$ forming one undeflected positive-parity image and $g$ negative-parity images located at the positions of each singularity (Petters et al. 2001).

Additionally, for isolated lenses with smooth $\psi\left(\boldsymbol{r}_{I}\right)$, the image

1 A lensing system is isolated if the time delay function $T_{\boldsymbol{r}_{S}}\left(\boldsymbol{r}_{I}\right)=\frac{1}{2} \mid \boldsymbol{r}_{I}-$ $\left.\boldsymbol{r}_{S}\right|^{2}-\psi\left(\boldsymbol{r}_{I}\right)$, which is the sum of the geometric and gravitational time delays, satisfies $T_{\boldsymbol{r}_{S}}\left(\boldsymbol{r}_{I}\right) \rightarrow \infty$ as $\left|\boldsymbol{r}_{I}\right| \rightarrow \infty$ for all finite, non-caustic $\boldsymbol{r}_{S}$. 

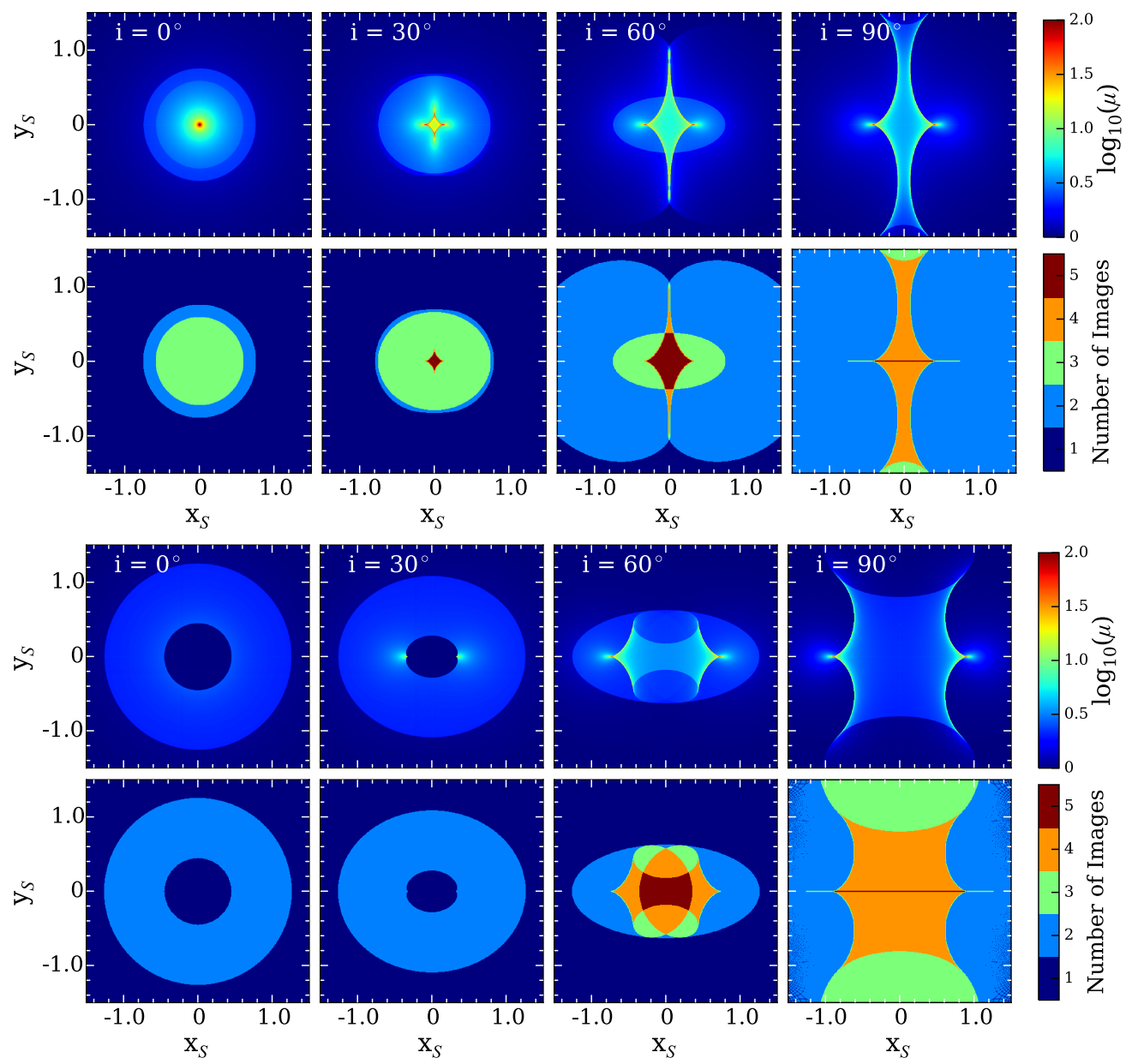

Figure 3. Magnification and image multiplicity maps for a ring-only lens. The top plot shows a ring of radius $a=0.75$ across a range of inclinations. The top row shows the magnification $\mu$ in the source plane, with the bottom row showing the number of images a source at a given position produces. The bottom plot is the same as the upper plot, but with the ring radius changed to $a=1.25$. Note that in both cases there are loci with finite change in magnification and with corresponding change in the number of images, which are pseudo-caustics discussed in the text.

multiplicity $m\left(\boldsymbol{r}_{S}\right)$ can be constrained to change when, and only when, the source crosses a caustic (Schneider et al. 1992). This can be seen by considering the map $\boldsymbol{r}_{S} \rightarrow m\left(\boldsymbol{r}_{S}\right)$, which associates each point $\boldsymbol{r}_{S}$ in the source plane with the total number of images formed by a point source located at $\boldsymbol{r}_{S}$. Since $m\left(\boldsymbol{r}_{S}\right)$ is an integer it cannot change continuously, and hence for smooth lens mappings $m\left(\boldsymbol{r}_{S}\right)$ can only change when the source crosses a caustic. Because of this, the caustic set must consist of closed curves (together with possible isolated point caustics). Indeed, if there were open caustic curves, a closed loop could be constructed in the source plane that had only one intersection with a caustic curve. A source traveling along this loop would then arrive back at its starting point with a different image multiplicity than it started with, which is impossible.

These constraints on image formation can be relaxed in systems, like the ones considered in this paper, whose lensing potential $\psi\left(\boldsymbol{r}_{I}\right)$ satisfies weaker differentiability or singularity conditions. Most relevantly for us, these constraints can be relaxed if $\psi\left(\boldsymbol{r}_{I}\right)$ is not twice-differentiable $\left(C^{2}\right)$. By Poisson's equation $\nabla_{\boldsymbol{r}_{I}}^{2} \psi\left(\boldsymbol{r}_{I}\right)=2 \kappa\left(\boldsymbol{r}_{I}\right)$, this is guaranteed if $\kappa\left(\boldsymbol{r}_{I}\right)$ is discontinuous, which occurs for the majority of the lensing geometries considered in this paper (although we consider the case of a $C^{\infty}$ mass distribution in Sec. 3.3). As was already shown in the face-on ring case and will be studied in more detail below, discontinuous mass distributions may allow for the existence of regions in the source plane across which the image multiplicity can change by one, violating the invariance of the image parity.

In the literature, curves in the source plane across which the image multiplicity changes by one are often called "pseudocaustics" (or "cuts" in e.g. Kovner 1987). Several previous studies have dealt with pseudo-caustics, primarily in the study of variants of singular isothermal spheres and ellipsoids (e.g. Wang \& Turner 1997; Evans \& Wilkinson 1998; Keeton et al. 2000; Rhie 2010), with which the ring+point lens shares several features. In most of these models, the pseudo-caustics appear as large smooth circles or ellipses. For example, the singular isothermal sphere model is defined by the lensing potential $\psi\left(\boldsymbol{r}_{I}\right)=R\left|\boldsymbol{r}_{I}\right|$ (implying $\left.\kappa\left(\boldsymbol{r}_{I}\right) \propto 1 /\left|\boldsymbol{r}_{I}\right|\right)$, and possesses a circular pseudo-caustic at $r_{S}=R$, where the number of images changes by one. As a source traveling from $r_{S}<R$ to $r_{S}>R$ crosses the pseudo-caustic, a negative-parity image "disappears" into the singularity at $r_{I}=0$. This is similar to what happens to the images of sources crossing the circular pseudocaustics in the face-on ring examples considered earlier, when a negative-parity image "disappears behind the ring" and becomes zero-sized.

In this paper, we adopt a slightly more general definition of pseudo-caustics, which is better suited for discussing the lensing 
systems we consider. We define pseudo-caustics to be curves in the source plane across which the magnification $\mu\left(\boldsymbol{r}_{S}\right)$ changes discontinuously, and yet remains finite. This definition is more general than the definition in terms of image multiplicities - besides including loci in the source plane where the number of images changes by one, it also includes curves across which the size of an image jumps sharply, which will be relevant in what follows.

The pseudo-caustics are found to possess a broad variety of morphologies across the parameter space of the lensing systems we consider. The formal caustics correspond to the degeneracy of the lensing Jacobian (implying $\mu\left(\boldsymbol{r}_{S}\right) \rightarrow \infty$ ), and as a consequence they are usually cusped. By contrast, the pseudo-caustics are defined to always have finite $\mu$, and they always appear to be smooth. In many cases, we will see that the existence of pseudo-caustics can lead to the presence of open caustics.

It is tempting to attribute the presence of pseudo-caustics in the thin ring case to the discontinuity of the lens mapping between the inner-ring and outer-ring solutions. However, the belt lensing systems we consider also exhibit a wide variety of pseudo-caustic features, even though the deflection angle $\boldsymbol{\alpha}_{b}\left(\boldsymbol{r}_{I} ; a_{i}, a_{o}, i\right)$ is continuous throughout the entire lens plane. Rather, we will see that it is the non-differentiability of the deflection angle (and hence the discontinuity of $\left.\kappa\left(\boldsymbol{r}_{I}\right)\right)$ that causes the formation of the pseudo-caustics.

Since the pseudo-caustics represent loci where $\mu$ is nondifferentiable, they can in principle be found by finding points in the source plane across which the magnification is discontinuous. However, the explicit analytic expression for $\left|\nabla_{r_{S}} \mu\right|$ is very unwieldy. In practice, we find the pseudo-caustics by using our inverse ray-shooting results to numerically compute the magnification map in the source plane and the associated image multiplicity map, which then allows us to find the pseudo-caustics by inspection.

As was the case for face-on rings and belts, we will see that the pseudo-caustics correspond to the boundaries of the belt/ring in the image plane mapped to the source plane, which allows us to derive an analytic expression for the pseudo-caustics (see Appendix C). Thus, for the models we consider, we can associate discontinuities in $\kappa\left(\boldsymbol{r}_{I}\right)$ with discontinuities in $\mu\left(\boldsymbol{r}_{S}\right)$, which define the pseudocaustics. We demonstrate this by explicitly mapping the belt/ring boundaries into the source plane, and checking that they correspond to regions in which $\mu\left(\boldsymbol{r}_{S}\right)$ is finite and discontinuous. We note that this is not a general proof that discontinuities in $\kappa\left(\boldsymbol{r}_{I}\right)$ are in a oneto-one correspondence with discontinuities in $\mu\left(\boldsymbol{r}_{S}\right)$, and that this correspondence may not hold for other types of lensing systems.

\subsection{Ring-Only Systems}

To proceed, we first turn our attention to the simplest case - that of a ring-only lensing system. Figure 3 shows magnification and image multiplicity maps for a range of ring parameters.

The top plot in Figure 3 shows a ring with radius $a=0.75$, while in the bottom plot the ring has $a=1.25$. In both cases, the inclination of the ring increases from left to right, going from face-on to edge-on. The top rows of each plot show magnification, while the bottom panels indicate the number of images for a source, $m\left(\boldsymbol{r}_{S}\right)$, at the given location in the source plane. We compute $m\left(\boldsymbol{r}_{S}\right)$ by sweeping over the image plane, identifying the set of all points $\left\{\left(x_{I}, y_{I}\right)\right\}$ that map to within a circle of radius $\epsilon \ll 1$ centred at $\boldsymbol{r}_{S}$, and then count the number of path-connected regions in the set $\left\{\left(x_{I}, y_{I}\right)\right\}$.

In the $a=0.75$ case (top plot), we see many boundaries between regions with different values of $m$, where the magnification has a finite jump. These are the pseudo-caustic curves mentioned above. In the face-on case (leftmost panel), from small to large radii, we see a circular $m=3$ region, an annular $m=2$ region, and finally the $m=1$ region, divided by the two pseudo-caustic curves. Examining our earlier results on face-on systems explains that the ring can have $m=3$ for small $\boldsymbol{r}_{S}$ because $a<1$. Applying equation (13) in the limit $a_{i}=a_{o}=a$ allows us to derive the radii of the pseudo-caustic circles as $\mathcal{P S}_{1}=a=0.75$ and $\mathcal{P S}_{2}=a^{-1}-a \approx 0.58$. The different image multiplicity regions can also be understood by plotting the solutions to the lens equation in a way similar to Figures 1 and 2 . There are three possible solutions, one (from $\boldsymbol{r}_{S}=\boldsymbol{r}_{I}$ ) corresponding to the undeflected light passing interior to the ring (the interior solution) and the other two (from $\boldsymbol{r}_{S}=\boldsymbol{r}_{I}\left(1-1 /\left|\boldsymbol{r}_{I}\right|^{2}\right)$ ) corresponding to the deflection by a central point with the same mass as the ring (the exterior solutions). The physically realized solutions are determined by the ring radius. For example, at $\left|\boldsymbol{r}_{S}\right| \gg 1$ only one of the exterior solutions is physical, giving rise to the $m=1$ region.

The solution for the $m=3$ region has one image from rays passing interior to the ring (hence undeflected), so the corresponding region in the image plane must be interior to the ring, which can be used to track the inclination of the ring. Indeed, as the ring becomes more inclined, the region becomes more elliptical (top plot of Fig.3). As the ring inclines, a $m=5$ diamond-shaped region forms near the centre and a vertical structure of increased magnification appears. Since the projected mass of the inclined ring becomes more and more concentrated toward the two ends of the major axis, the normal caustic feature exhibits some similarity to that from an equal-mass binary lensing system with the two point masses symmetrically placed on the major axis, with the positions approaching $\pm a$ as the ring becomes edge-on. That is, as the inclination increases, the caustic features mimic those of close- and intermediate-separation equal-mass binary point mass (e.g. Schneider \& Weiss 1986; Liebig et al. 2015) and binary isothermal sphere (Shin \& Evans 2008) lenses. Additionally, the combination of a large circular pseudo-caustic and a central asteroidal caustic seen in the $i=30^{\circ}$ panel is similar to the caustic structures of singular isothermal sphere potentials and variants thereof (see e.g. Figure 1 of Rhie 2010 and Figure 5 of Kovner 1987).

The case with $a=1.25$ (bottom plot) is fairly different. When seen face-on, the ring is no longer concentrated enough to form multiple images near the centre, since the entire mass distribution of the ring lies outside of its own Einstein radius. The possible physical solution to the lens equation includes that from the undeflected rays and that corresponding to the case of a point-mass lens (the one with the image outside the Einstein ring radius), with the former and latter applying to small and large radii, respectively. In between, there is an annular region where both apply, and the boundaries give the two pseudo-caustic curves, which are circles of radii $\mathcal{P S} \mathcal{S}_{1}=a=1.25$ and $\mathcal{P S} \mathcal{S}_{2}=a-a^{-1}=0.45$. As the ring becomes inclined, this annular region pinches down about the major axis, and two folded regions of high magnification appear at the inner parts of the annulus. As the inclination continues to increase, regions with higher image multiplicities start to form. As with the $a=0.75$ case, the normal caustics share similarities with those from an intermediate-separation equal-mass binary lens. For both values of $a$, we see the appearance of a narrow high- $m$ region located along the $x_{S}$ axis. For a point source the additional images formed in this region occur when the $y_{S}$-coordinate of the source is strictly zero, and so the corresponding high- $m$ region has zero thickness.

We now turn our attention to the ring+point mass system and provide an illustrative understanding of the pseudo-caustics and 
open caustics. Finally, we present the results for the belt+point mass system.

\subsection{Ring/Belt+Point Mass Systems}

\subsubsection{Ring+Point Mass System}

With a point mass placed at the centre of the ring/belt, the solutions become more complex. Examining the critical curves in the image plane and the corresponding caustic curves in the source plane is helpful for us to understand the lensing properties, especially the pseudo-caustics and open caustics.

The thick black curves in Figure 4 are the critical curves (top plots) and the corresponding caustic curves (bottom plots) for the ring+point mass system. To understand these curves, it is useful to present the full sets of critical and caustic curves for the interior and exterior solutions, which are shown as the blue and red curves, respectively. For the interior solution, the term "full" refers to the curves determined from the centre point mass, as if the ring did not exist. For the exterior solution, "full" means that we plainly apply the exterior solution even if the rays pass interior to the ring. Or effectively, we shrink the projected ring confocally to a degenerate ellipse with zero minor axis so that the exterior solution can be extended to small radii. We investigate which parts of the critical curves are allowed to form the final critical curves (thick black) given the configuration of the system, and deduce the pseudo-caustics and open caustics. For the system shown in Figure 4 , the ring and point mass have equal masses, with the ring having a radius of either $a=0.75$ or $a=1.25$ as labelled in the panels. The inclination of the ring increases from $30^{\circ}$ to $90^{\circ}$ from left to right. The scales are normalized to the Einstein ring radius of the combined ring+point mass system.

The "full" critical curve for the interior solution is simply a circle with radius $1 / \sqrt{2}$, the Einstein ring for the centre point mass (since we normalize by the Einstein ring radius for the total ring+point mass, which is twice the point mass here). The corresponding caustic is the point at the origin. For the exterior solution, the "full" critical curve is also a circle (the Einstein ring for the total ring+point mass) for the system at face-on. As the ring becomes more inclined, its mass distribution approaches more toward a binary lens with the two equal point masses symmetrically placed on the $x$-axis and on the two sides of the centre point mass toward $( \pm a, 0)$. The whole system mimics the one composed of three point masses (Daněk \& Heyrovský 2015). For the $a=0.75$ case, besides a big ring-like critical curve, four small ringlet-like critical curves are formed. The big and small curves correspond to the central diamond-shaped caustic and the four planetary cusp caustics, respectively. As a whole, at high inclination, the critical and caustic curves share similarities with the close-separation triple point lensing system. For the $a=1.25$ case, the analysis is similar and at high inclination we find the system to be similar to the intermediate- or wide-separation triple point lens.

With the "full" critical and caustic curves in place for both the interior and exterior solutions, we now analyse which parts of the curves are physically realized by considering the validity of the solutions. Unlike in Section 3.1 where we explain things in the source plane, we find it is more intuitive to work in the image plane, since the validity of the solutions is determined by the image position (whether it is interior or exterior to the ring) and since the projected ring is also naturally described in the image plane.

The dashed black curve in each critical-curve panel of Figure 4 delineates the projected ring. For the interior solution, images that form outside of the projected ring are not physical. Therefore the part of the critical curve outside the ring (indicated by thin blue lines) is not allowed. The allowed lens-plane area for the interior solution becomes smaller as the ring becomes more inclined. The corresponding caustic is still the point at the origin of the source plane.

For the exterior solution, the situation is reversed. The part of the critical curve inside the ring (indicated by thin red lines) is not allowed. In a few cases (e.g. with $a=0.75$ and $i=60^{\circ}$ ), the four small ringlet-like critical curves are inside the ring and hence are not allowed, which leads to the disappearance of the "planetary" caustics. For the case with $a=1.25$ and $i=60^{\circ}$, the allowed part of the critical curve forms four separated arclets, as a result of the truncation imposed by the ring. They lead to four separated caustic curves, and the truncation in the critical curve makes the caustic curve become open.

Since it is the projected position of the ring that determines which solution is valid, the pseudo-caustics can be obtained by mapping the projected ring onto the source plane, according to the interior and exterior solution, respectively. The inner (outer) boundary of the mapped ring is shown as purple (green) curves in the source plane that result from mapping the projected ring with the interior (exterior) solution in the caustic-curve panels of Figure 4. Formal caustics can become open as they intersect the mapped ring. Since the mapping properties change discontinuously as the impact point (image) crosses the ring, the curves of the mapped ring are just the pseudo-caustics mentioned before. Notice that each pseudo-caustic is smooth, since the lens mapping is continuous just within and just outside the ring (which also holds for the thick belt case). Since the magnification is finite at the pseudo-caustics, magnification and image multiplicity maps must be constructed to study them.

Figure 5 shows the magnification and image multiplicity maps for the ring+point systems shown in Figure 4, with $q=1$. Clearly, the main features in the magnification map correspond closely with the formal caustics and pseudo-caustics in Figure 4.

For $a=0.75$ (top plot), when seen face-on, we see two pseudo-caustics, similar to the ring-only case. However, the regions inside the pseudo-caustics have higher image multiplicities than the ring-only case. The reason is that the presence of the centre point mass allows the maximum number of solutions to be four, opposed to three as in the ring-only case. Using our earlier results for faceon belt+point mass systems and taking $a_{i}=a_{o}=a$, we can derive the radii of the pseudo-caustic circles to be $\mathcal{P} \mathcal{S}_{1} \approx 0.11$ and $\mathcal{P S}_{2} \approx 0.82$. As the inclination increases, the three-image region becomes larger and more oblate. Interestingly, two lobes of $d e-$ creased image multiplicity appear within the $m=3$ region. When seen edge-on, these regions become larger and push out the $m=3$ regions to larger radii.

In the bottom plot of Figure 5, the ring is enlarged to have a semi-major axis of $a=1.25$. The pseudo-caustics in this case appear distinct from the $a=0.75$ case. When seen face-on, we see an $m=3$ annular region bounded by the two pseudo-caustics. When the ring inclines, the annulus folds down over itself, creating complicated overlapping features (e.g. in the panel with $i=60^{\circ}$ ) from the interplay between the pseudo-caustics and the formal caustics. When seen edge-on, $m=1$ regions and small $m=7$ regions are formed. Although edge-on $a=0.75$ and $a=1.25$ ring+point mass systems are very similar geometrically, they approximately correspond to close- and intermediate-separation triple point mass lensing systems and hence the magnification maps are different. 

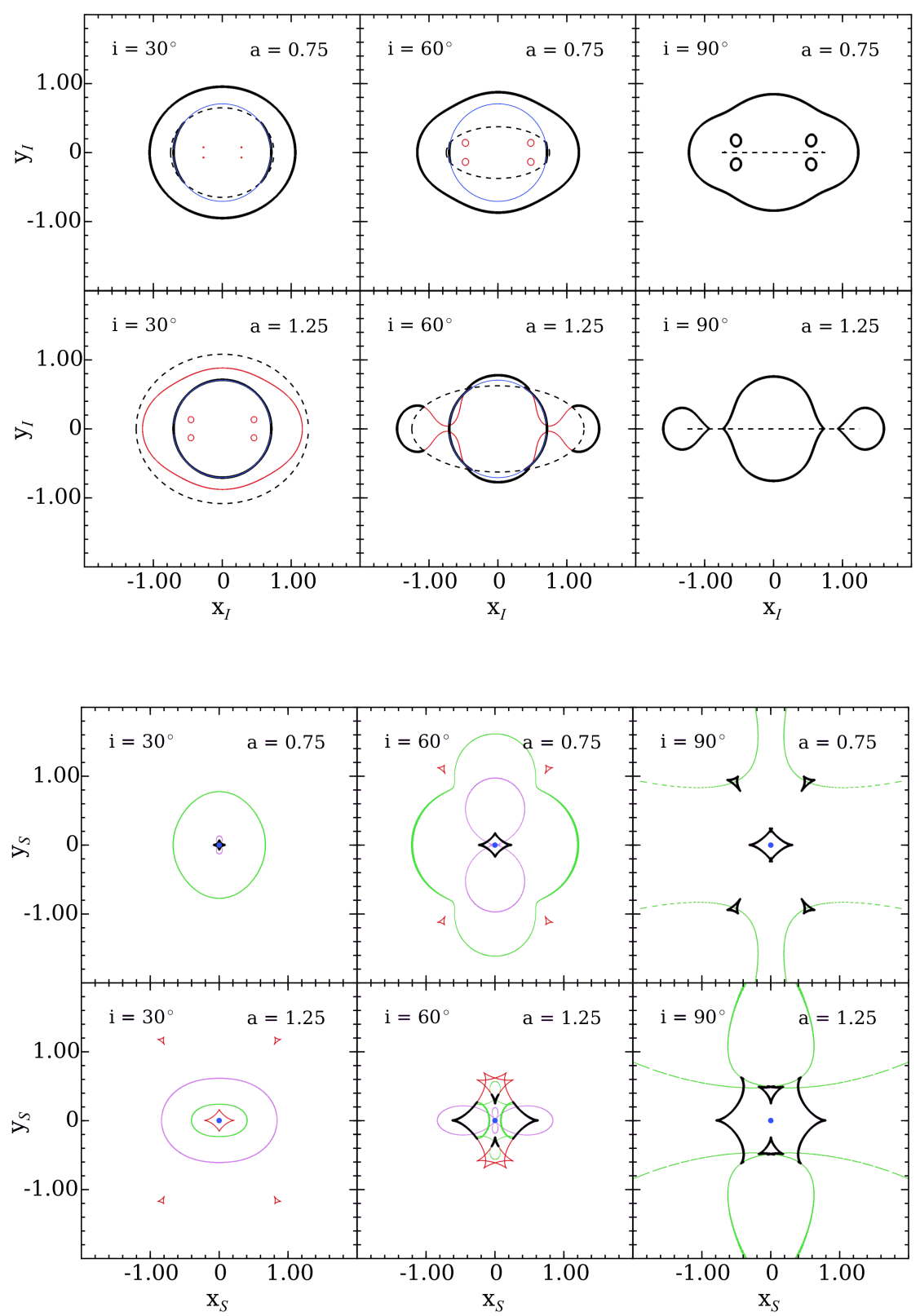

Figure 4. Critical curves (top plot) and caustic curves (bottom plot) for a ring+point lens. The ring and the point mass have equal masses. The radius and inclination of the ring are labelled in each panel. In each critical-curve panel, the blue (red) thin curves connected with the thick solid ones form the "full" critical curves from the interior (exterior) solution. The dashed curve delineates the projected ring in the image (lens) plane. The critical curves from the interior (exterior) solution becomes invalid for the parts outside (inside) the ring. The final allowed critical curves are in thick black. The corresponding caustics are denoted with the same colour and line types in the caustic-curve panels. The ring is mapped onto the source plane according to the interior (purple) and exterior (green) solution, respectively. These mapped ring curves form the pseudo-caustics. See the text for detail.

\subsubsection{Belt+Point Mass Systems}

We now widen the ring to a belt, and study the resulting effects on the morphology of the pseudo-caustics and the general magnification pattern. The lensing solutions are affected by the projected mass density (i.e. the width of the belt), as seen in Figure 1. For the following examples, we fix the belt width to be $\Delta a=0.3$. In the belt+point mass case, the pseudo-caustics correspond to the inner and outer edges of the belt mapped onto the source plane accord- ing to the interior and exterior solutions, respectively. The explicit expression for the pseudo-caustics can be found in Appendix C.

Figure 6 shows the magnification and image multiplicity maps for belts with different mean radii. With $\Delta a=0.3$, the belt radius is centred at $a_{c}=0.75$ in the top plot and $a_{c}=1.25$ in the middle plot. In the bottom plot it is situated so that half of its mass lies at $a>1$ and the other half lies at $a<1$. In all plots, we take $q=1$.

For $a=0.75$, we only see two images formed for the face-on case since $a_{o}<a_{o, \text { cr }}$, and the surface density of the belt is not high enough to generate additional images, similar to the case indicated 

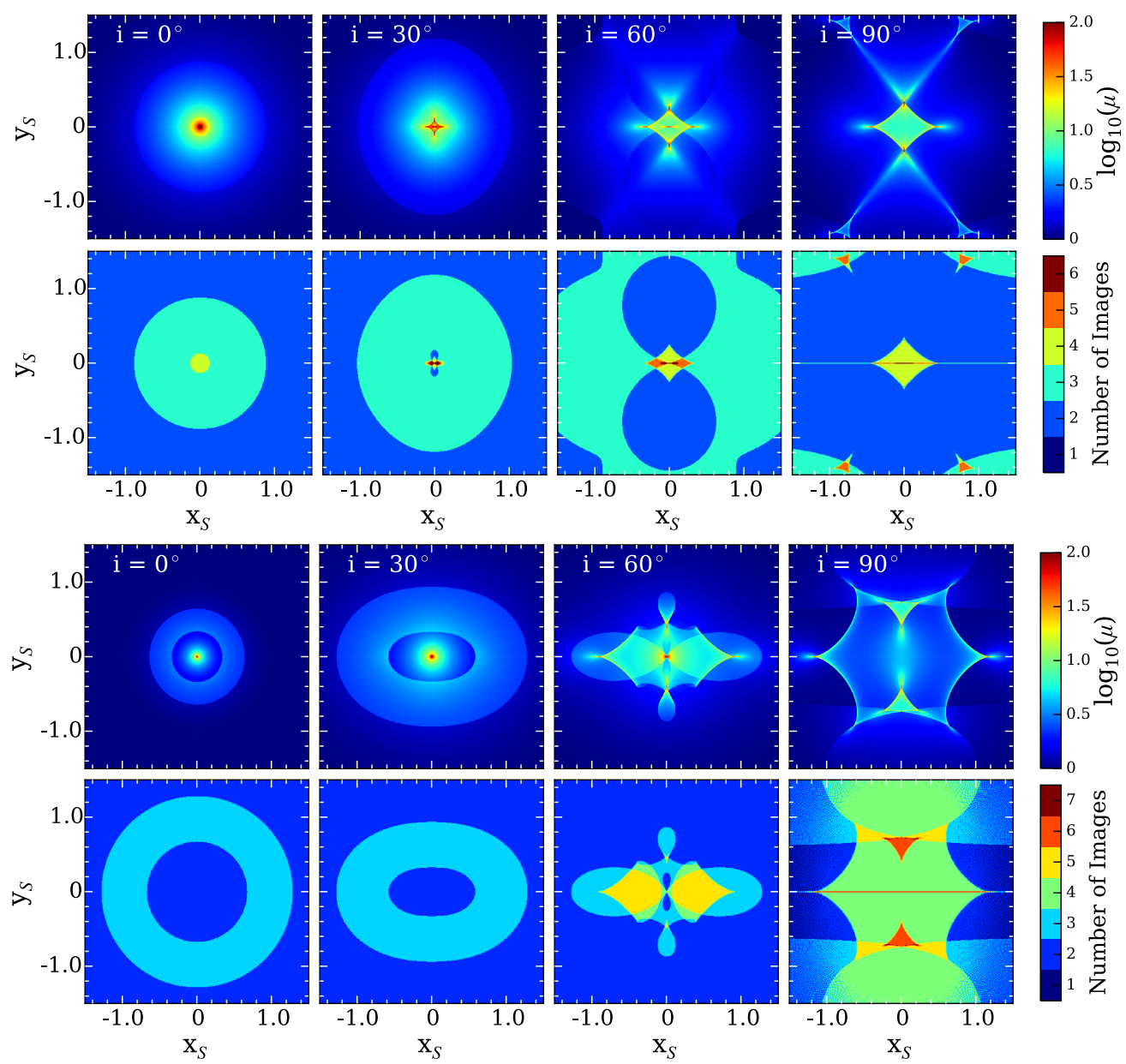

Figure 5. Magnification and image multiplicity maps for a ring+point lens. The ring and point mass have equal masses. The ring has radius $a=0.75$ in the top plot and $a=1.25$ in the bottom plot. As in Figure 3 there exist pseudo-caustics associated with changes in the image multiplicity. See Figure 4 for the corresponding pseudo-caustics associated with the cases with $i \geqslant 30^{\circ}$.

by the blue curve in Figure 1. However, we see a ring-like structure present in the magnification map, with no correspondent image multiplicity change. This ring-like structure corresponds to rays passing through the belt, experiencing deflection from both the belt and the centre point mass. This leads to the change in the slope of the $r_{S}-r_{I}$ relation (as illustrated in Figure 1). This means that one of the images becomes larger in size, resulting in an overall increase in magnification $\left(d r_{I}^{2} / d r_{S}^{2}\right)$. This example shows that pseudo-caustics need not to be associated with changes in the image multiplicity they can also correspond to discontinuous changes in the size of an image.

When the belt inclines, a narrow $m=4$ annular region bounded by pseudo-caustics appears that evolves into a fan-shaped region at $i=60^{\circ}$. When the belt is seen edge-on, the magnification map is similar to the case of the $a=0.75$ edge-on ring (right panels in the top plot of Figure 5), as both approximately approach the close-separated triple point lensing system.

For the belt with radius centred at $a=1.25$ (middle plot of Figure 6), the magnification pattern shares some broad similarities with that of the $a=1.25$ ring case (bottom plot of Figure 5). The main noticeable differences are that at low inclination the belt+point case has a narrower region bounded by pseudo-caustics and that in the face-on case, there is no image number change when crossing the pseudo-caustics (for the same reason as in the above $a=0.75$ case). In particular, at edge on, the three cases from $a=0.75$ to $a=1.25$ are similar to the triple point lensing system going from close toward intermediate separations. The overall features for the case with half mass inside and half mass outside the Einstein ring radius are in between the $a=0.75$ and $a=1.25$ cases.

\subsubsection{Examples of Images from the Ring+Point System}

In Figure 7, we provide examples of the sorts of images formed for sources lensed by ring+point mass systems. The top plot shows the case of a face-on ring with $a=1.25$. The left panel shows the image multiplicity map with the red and blue circles representing two sources in the source plane, and the corresponding images are shown in the image plane in the right panel. The red source is mapped to two images by the centre point mass, while the blue source inside the region bounded by the pseudo-caustics is able to pass rays outside the ring (black circle) and take advantage of the ring's lensing potential to form three images, with the third appearing just outside the ring.

The bottom plot considers a slightly more complicated case, with the ring inclined to $i=60^{\circ}$. Even though the image multiplicity map in the left panel looks complicated, the way in which each source is lensed is easy to understand. The red source is lensed by 

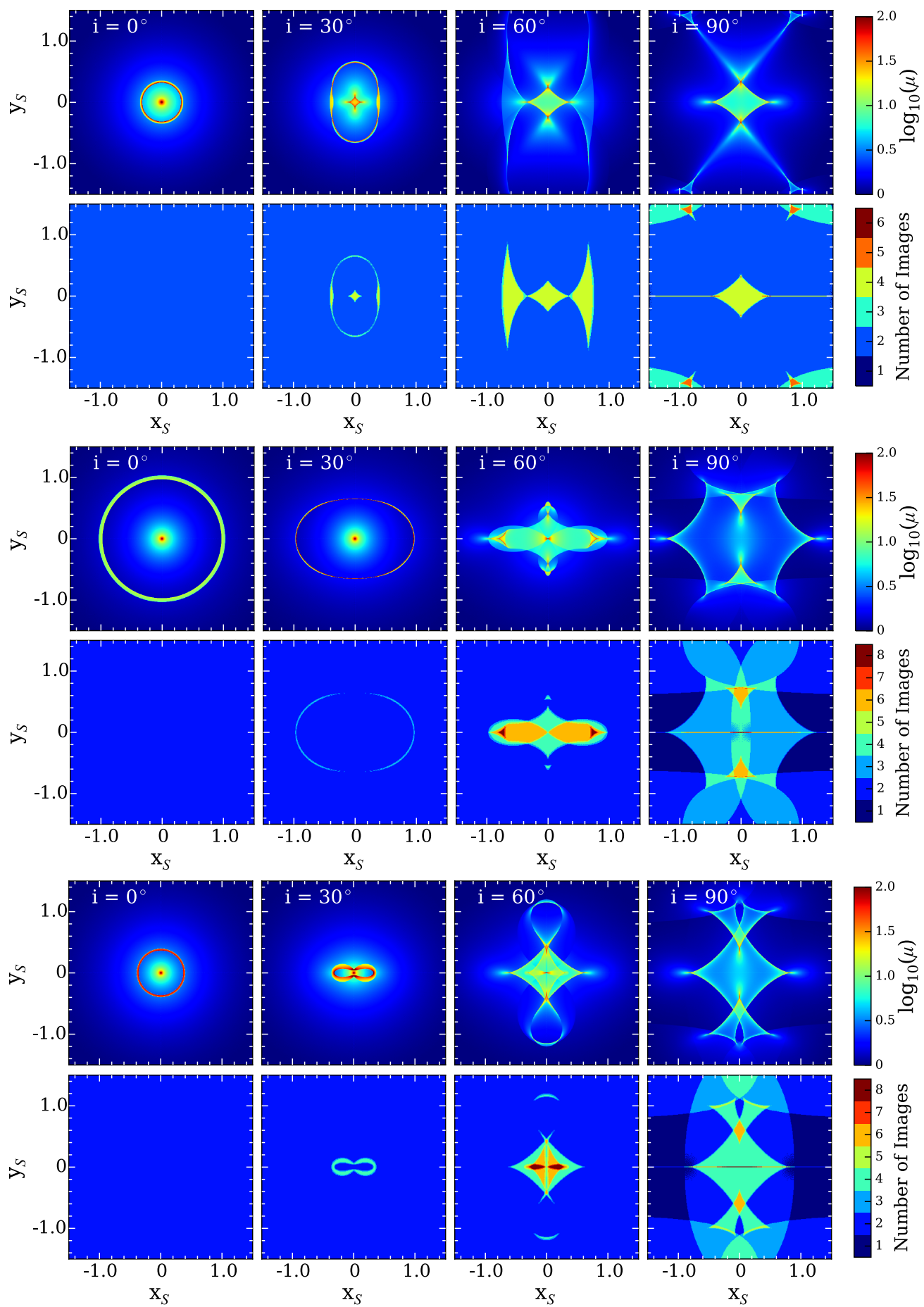

Figure 6. Magnification and image multiplicity maps for a belt+point mass lens at different belt inclinations. In each plot, the belt has a width of $\Delta a=0.3$. In the top plot, the radius of the belt is centred at $a_{c}=0.75$, in the middle plot it is centred at $a_{c}=1.25$, and in the bottom plot the belt is situated so that half of its mass is at $a<1$ and the other half is at $a>1$. In all the cases, there exist pseudo-caustics associated with a discontinuous change in magnification and a corresponding change in the number or size of images.

the centre point mass, but only one of the two possible images is inside the ring and hence is physical. The two images outside the ring come from rays deflected by both the point mass and the ring. The blue source can create two images inside the ring from deflection solely by the centre point mass. On the $x_{I}$ axis, it can use additionally the ring's deflection to create an image just outside the ring. Furthermore, it can create two additional images outside the ring by taking advantage of the $y_{I}$ component of the deflection. In total, five images emerge for this source. 

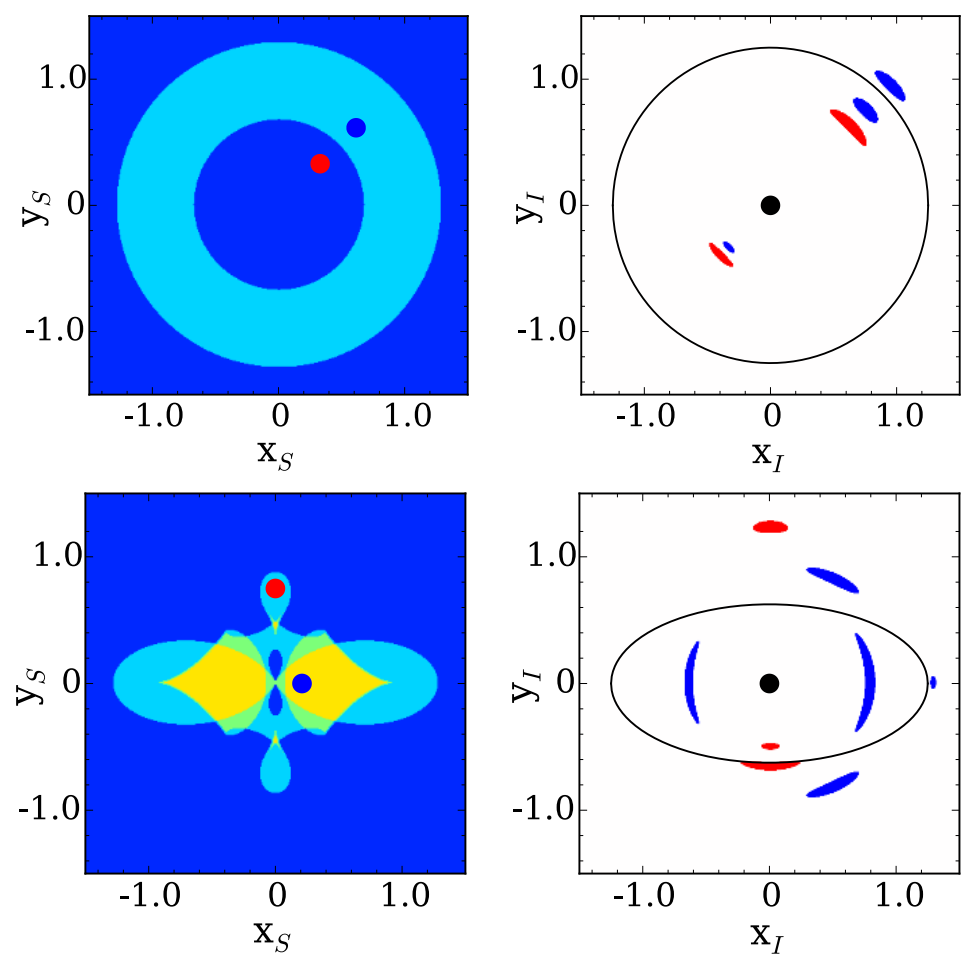

Figure 7. A demonstration of the lens mapping for two sample sources for a ring+point lens with the ring and the point having equal mass. The ring has a radius $a=1.25$ and is seen face-on for the top panels and inclined (with inclination angle $i=60^{\circ}$ ) for the bottom panels. The left panels show image multiplicities in the source plane, with two sources plotted as the red and blue circles. The right panels show how these sources appear in the image plane, with the ring+point system drawn in black.
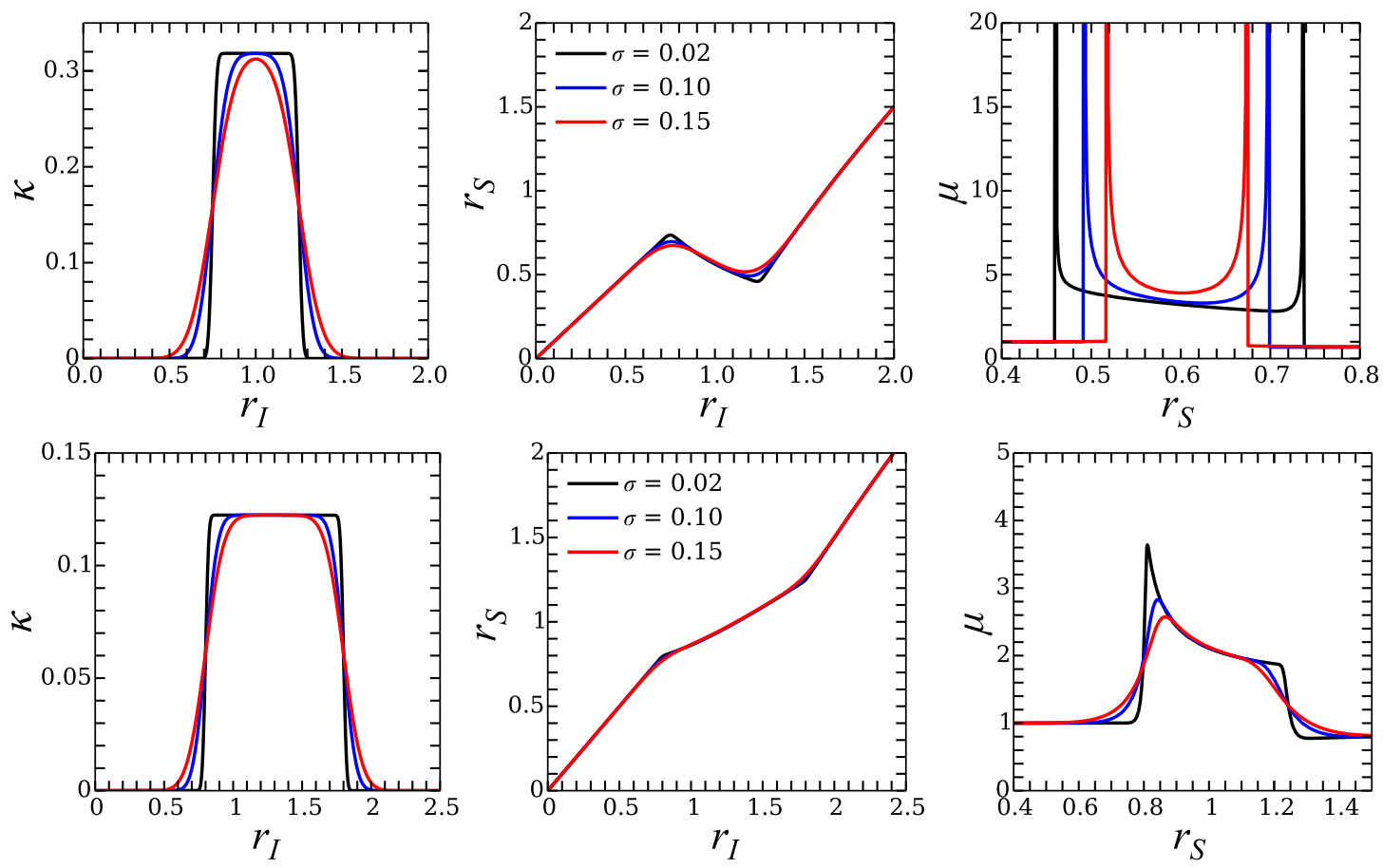

Figure 8. The effects of smoothing out the mass distribution for face-on belts. From left to right, the columns show the surface density profile $\kappa\left(r_{I}\right)$ of the belt, the solution to the lensing equation $\left(r_{S}-r_{I}\right.$ mapping), and the magnification $\mu\left(r_{s}\right)$ for three different values of the smoothing parameter $\sigma$. In the top rows, the belt has $a_{c}=1$ and $\Delta a=0.5$, so that $\Delta a<(\Delta a)_{\mathrm{cr}}$, meaning that the belt can form multiple images. The pseudo-caustics (associated with image number change) in the limit of $\sigma \rightarrow 0$ for such a case originates from disappearing formal caustics. In the bottom row the belt has $a_{c}=1.3$ and $\Delta a=1$ so that $\Delta a>(\Delta a)_{\mathrm{cr}}$, and the belt forms a single image for all $r_{S}$. The pseudo-caustics (associated with image size change) in the limit of $\sigma \rightarrow 0$ for such a case comes from sharpening the edge of the bump in the magnification map. See the text for detail. 


\subsection{Smooth Density Distributions and the Origin of Pseudo-Caustics}

The belt/ring+point systems considered so far have all possessed discontinuous mass distributions. This allows for a simple analytic treatment of such systems, but in order to connect our results with potential astrophysical applications (namely microlensing events caused by asteroid belt + star lenses), we need to consider a more realistic smoothened mass distribution. Working with a smooth mass distribution means that the conditions necessary for applying the relevant theorems from singularity theory regarding image multiplicities and caustic morphologies are satisfied, and as such we expect the pseudo-caustics features to change. As we will see, by smoothing out the mass distribution, pseudo-caustics either change into formal caustics or become smeared into regions of finite but smooth magnification jumps, with the former corresponding to pseudo-caustics associated with image number change and the latter with image size change. This provides a clear picture of the origin of the pseudo-caustics encountered in the systems considered in this paper with discontinuous mass distributions.

To illustrate the effects of smoothing, we analyze a simple face-on belt-only model with a smoothed mass distribution of the form

$\kappa\left(r_{I}\right)=\frac{1}{N}\left[\operatorname{erf}\left(\frac{r_{I}-a_{i}}{\sigma}\right)-\operatorname{erf}\left(\frac{r_{I}-a_{o}}{\sigma}\right)\right]$,

where $N$ normalizes the distribution. It represents a belt of characteristic inner radius $a_{i}$ and outer radius $a_{o}$, with the parameter $\sigma$ controlling the smoothness of the inner and outer edges. The belt systems studied in previous sections correspond to the limit of $\sigma \rightarrow 0$.

The precise effect of the smoothing on the pseudo-caustics depends on the size of the smoothing parameter $\sigma$ and the ratio $\Delta a /(\Delta a)_{\mathrm{cr}}$, where $(\Delta a)_{\mathrm{cr}}=2 /\left(a_{o}+a_{i}\right)$ [equation (12)] is the critical belt width. As discussed earlier, sharp belts with $\sigma=0$ and $\Delta a>(\Delta a)_{\text {cr }}$ can generate pseudo-caustics that result in changes in the sizes of images. If the width of the belt satisfies $\Delta a<(\Delta a)_{\mathrm{cr}}$, the belt can produce pseudo-caustics that correspond to changes in the image multiplicity. In these two regimes, the effects of smoothing are qualitatively different. This is illustrated in Figure 8. In each column of the plot, from left to right the three panels show the surface density profile of the belt, the solution to the lensing equation $r_{S}\left(r_{I}\right)$, and the magnification as a function of $r_{S}$, respectively, for three values of $\sigma(0.02,0.10$, and 0.15$)$.

In the top three panels, the belt has $\Delta a<(\Delta a)_{\mathrm{cr}}$. The smooth mass distribution of the belt leads to a smooth $r_{S}\left(r_{I}\right)$ mapping, and the pseudo-caustics associated with a change in image multiplicity transform into circular formal caustics with divergent $\mu$. As $\sigma$ increases, the two formal caustics move closer together in the source plane and the characteristic width of the caustic feature (defined as the extent in the source plane with magnification $\mu$ above some large threshold value) increases. Conversely, as we decrease $\sigma$, the extent of the caustic feature shrinks. In the limit of $\sigma \rightarrow 0$ it shrinks to zero and the caustic feature formally disappears. In this limit, the magnification shows a sharp finite jump at the position of each former caustic, and the feature is identified as a pseudo-caustic. So in the $\Delta a<(\Delta a)_{\text {cr }}$ regime, the pseudo-caustics are in fact associated with infinitesimally small formal caustics.

In the bottom three panels, the belt is wide, with $\Delta a>(\Delta a)_{\mathrm{cr}}$. When $\sigma \rightarrow 0$, the system possesses pseudo-caustics associated with image size changes induced by sharp changes in $\partial r_{S} / \partial r_{I}$, which create finite jumps in $\mu$. Smoothing the mass distribution softens the jumps in $\mu$, and the pseudo-caustics become smeared out. We can thus think about the origin of the pseudo-caustics in the following way: for a smooth mass distribution with $\sigma \ll \Delta a$, $\partial r_{S} / \partial r_{I}$ changes smoothly over small annular regions surrounding the belt boundaries. These changes translate to smooth magnification jumps within narrow annular regions of the source plane. As $\sigma \rightarrow 0$, these jumps in $\mu$ become sharp while remaining finite. The loci where the finite jumps occur are identified as pseudo-caustics.

\section{SUMMARY AND DISCUSSION}

In this paper, we have explored the gravitational lensing properties of a lens made of a circular ring/belt, with and without a centre point mass. These systems exhibit complex features that vary significantly within the parameter space of the ring/belt, including open caustics and pseudo-caustics. We detail a few examples that illustrate these features.

The key property of a circular ring/belt lenses is that the rays passing interior to the projected ring/belt are not deflected by the ring/belt, while those passing exterior to the projected ring/belt are deflected. We can thus consider two types of solutions to the lensing equation - one involves deflection from the entire ring/belt system, which we call the exterior solution, and one involves no deflection from the ring/belt, which we call the interior solution. The projected ring/belt thus acts like a filter to select which one of these solutions is applicable at different points in the image plane. That is, in the image plane, the ring/belt sets the boundary to determine which images are physical. Some solutions are forbidden, because they are obtained as interior solutions but correspond to rays passing exterior to the ring/belt, or vice versa. Because of this selection process, for the critical curves associated with the exterior solutions, only the part exterior to the belt/ring remains, and similarly for those associated with the interior solutions. This allows for broken critical curves, and hence open caustics.

In the image plane, the ring/belt is the region that connects the interior and exterior solutions. The connection can be continuous in the belt case and discontinuous in the thin ring case. In either case, the joint solution to the lens equation is generically nondifferentiable at the boundaries of the belt/ring. Since in general the boundaries of the belt/ring are not related to the critical curves, the boundaries are not associated with the singularities in the lensing mapping. The corresponding curves in the source plane (obtained by mapping the belt/ring boundaries into the source plane) are not associated with formal caustics (which have infinite magnification), but although there exists a finite discontinuity in magnification across such curves. As such, they are dubbed "pseudocaustics". Such a magnification change can be associated either with a change in the number of images formed by a source (e.g. for thin rings or narrow belts) or not (e.g. in the case of an extended belt; see the example in Fig. 1). The magnification change in the latter case is caused by a sharp change in the size of an image. These effects are present because of the discontinuous nature of the mass distribution of the ring/belt. When the density distribution is smoothened, the pseudo-caustics become formal caustics if the belt is narrow, and disappear (or more precisely, the finite magnification jumps associated with the pseudo-caustics become "smeared out") if the belt is wide. That is, the pseudo-caustics associated with changes in image multiplicity are attributed to infinitely-thin formal caustics, and those associated with sharp changes in the size of an image are attributed to the sharpening of the edges of a smooth bump in the magnification map.

The images modified by the boundaries of ring/belt in the im- 
age plane and the magnification patterns modified by the pseudocaustics in the source plane form the main lensing properties of the lenses considered in this paper. We see a complex interplay between the ring/belt and the critical curves in the image plane, or between the pseudo-caustics and the formal caustics in the source plane. Usually, analysing the (formal) caustics of a lensing system provides enough information to determine the image multiplicities, the general magnification patterns, and the light curve properties expected for a given source trajectory. However, once a ring/belt is introduced to the lensing system, in addition to the formal caustics, an analysis of the pseudo-caustics is needed to obtain a full picture of the lensing properties of the system. A vast amount of information hidden at finite magnifications would be missed if a study concerning such systems were to focus only on the formal caustics. Our work thus serves as a cautionary tale for investigations into other novel lensing geometries.

The deflection and magnification of the lens mappings we have considered can be computed analytically, and the pseudocaustics can be easily found by mapping the edges of the ring/belt to the source plane. However, finding analytic solutions to the lensing equation is fairly tedious with our current approach, and we have been forced to restrict our analytic analysis to the simplest cases of face-on and edge-on systems (for a discussion of the latter, see Appendix A). Additionally, we lack an analytic expression for the image multiplicity at an arbitrary point in the source plane, which currently must be found numerically. A more general mathematical understanding of the connection between pseudo-caustics and image multiplicities would be of great utility to the theoretical study of gravitational lensing.

Although our analysis is mainly from a theoretical viewpoint and aims at understanding the main features in a lensing system with rings/belts, there are also potential applications of our work to astronomical situations. In a related paper (Lake, Zheng, \& Dong 2016), we discuss the possibility of detecting extrasolar asteroid belts based on their microlensing signatures. For the lensing system of interest, the main difference lies in the mass ratio of the belt to the centre point (star) - realistic mass ratios are around $q \sim 10^{-6}$ or smaller, as opposed to the $q=1$ case considered here. However, it is shown that the pseudo-caustics still have a significant effect on the magnification maps. This gives rise to interesting features in microlensing light curves that can potentially be used to detect extrasolar asteroid belts.

The other possible application is the lensing by ring galaxies. As a result of head-on collisions between a disk galaxy and another galaxy (e.g. Lynds \& Toomre 1976; Appleton \& StruckMarcell 1996), a radially expanding density wave is excited in the disk galaxy, which triggers star formation. The fraction of stellar mass resultant from the star formation is at the sub-percent level (e.g. Fogarty et al. 2011), and the total perturbed mass in the ring can be even higher. If a background source (galaxy or quasar) happens to be lensed by a ring galaxy, the observation of such a lensing system can be used to constrain the mass in the star-forming ring, which can be used to test the density wave model of star formation and aid the understanding of ring galaxies.

\section{ACKNOWLEDGEMENTS}

We thank the anonymous referees for constructive comments and suggestions that helped improve the paper. The support and resources from the centre for High Performance Computing at the University of Utah are gratefully acknowledged.

\section{REFERENCES}

Appleton, P. N., \& Struck-Marcell, C. 1996, Fundamentals Cosmic Phys., 16,111

Batista, V., Beaulieu, J.-P., Gould, A., et al. 2014, ApJ, 780, 54

Bond, I. A., Udalski, A., Jaroszyński, M., et al. 2004, ApJ, 606, L155

Blandford, R. D., Saust, A. B., Brainerd, T. G., \& Villumsen, J. V. 1991, MNRAS, 251, 600

Bradač, M., Treu, T., Applegate, D., et al. 2009, ApJ, 706, 1201

Chandrasekhar, S. 1969, Ellipsoidal figures of equilibrium

Einstein, A. 1936, Science, 84, 506

Erdl, H., \& Schneider, P. 1993, A\&A, 268, 453

Fogarty, L., Thatte, N., Tecza, M., et al. 2011, MNRAS, 417, 835

Lake, E., Zheng, Z., \& Dong, S. B. 2016, arXiv:1601.03052

Liebig, C., D’Ago, G., Bozza, V., \& Dominik, M. 2015, MNRAS, 450, 1565

Lynds, R., \& Toomre, A. 1976, ApJ, 209, 382

Mao, S., \& Paczynski, B. 1991, ApJ, 374, L37

Petters, A. O., Levine, H., \& Wambsganss, J. 2001, Singularity theory and gravitational lensing, Vol. 21 (Springer Science \& Business Media)

Rhie, S. H. 2010, ArXiv e-prints, arXiv:1006.0782

Schneider, P., \& Weiss, A. 1986, A\&A, 164, 237

Schneider, P., Ehlers, J., \& Falco, E. E. 1992, Gravitational Lenses

Schramm, T. 1990, A\&A, 231, 19

Tyson, J. A., Kochanski, G. P., \& Dell'Antonio, I. P. 1998, The Astrophysical Journal Letters, 498, L107

Vikram, V., Chang, C., Jain, B., et al. 2015, ArXiv e-prints, arXiv: 1504.03002

Wittman, D. M., Tyson, J. A., Kirkman, D., Dell'Antonio, I., \& Bernstein, G. 2000, Nature, 405, 143

Bourassa, R., \& Kantowski, R. 1975, ApJ, 195, 13

Bray, I. 1984, MNRAS, 208, 511

Burke, W. L. 1981, ApJ, 244, L1

Daněk, K., \& Heyrovský, D. 2015, ApJ, 806, 99

Evans, N. W., \& Wilkinson, M. I. 1998, MNRAS, 296, 800

Hoekstra, H., Bartelmann, M., Dahle, H., et al. 2013, Space Sci. Rev., 177, 75

Keeton, C. R., Mao, S., \& Witt, H. J. 2000, ApJ, 537, 697

Kovner, I. 1987, ApJ, 312, 22

McKenzie, R. H. 1985, Journal of Mathematical Physics, 26, 1592

Petters, A. O. 1995, Journal of Mathematical Physics, 36

Shin, E. M., \& Evans, N. W. 2008, MNRAS, 390, 505

Tessore, N., \& Metcalf, R. B. 2015, A\&A, 1507.01819

Wang, Y., \& Turner, E. L. 1997, MNRAS, 292, 863

\section{APPENDIX A: ANALYSIS OF EDGE-ON RINGS}

While a general analytic analysis for the inclined systems considered in the text is possible, the equations quickly become unwieldy. Thus, it is helpful to study the limiting cases of edge-on and faceon systems analytically in order to build an intuition for how the general cases behave. In this appendix, we present a more quantitative understanding for how edge-on rings behave as lenses, having treated the simpler face-on case in the main text (Section 2.1). For simplicity, we only consider sources located along either the $x_{S}$ or $y_{S}$ axes.

First, let us assume a source located along the $y_{S}$ axis, with $\boldsymbol{r}_{S}=\left(0, y_{S}\right)$ for $y_{S}>0$. There are two types of images - those located along the $y_{I}$ axis $\left(\boldsymbol{r}_{I}=\left(0, y_{I}\right)\right)$, and those with a nonzero $x_{I}$ component. Starting from equation (19), we can derive that the images along the $y_{I}$ axis are found by solving

$\left(y_{I}-y_{S}\right)^{2}\left(y_{I}^{2}+a^{2}\right)-1=0$.

This equation generically has two real solutions for $y_{I}$. For small $y_{S}$ (more precisely, for $y_{S}<1 / a$ ), there are both positive and negative solutions for $y_{I}$, corresponding to rays passing "over" or "under" 
the ring. As $y_{S}$ increases past the critical value of $1 / a$, the "under the ring" solution occurs at $y_{I}>0$, and hence becomes unphysical.

The other images with $x_{I} \neq 0$ are found by solving the equation

$1-y_{S} / y_{I}=\left(1-y_{I} / y_{S}\right)^{2}\left(a^{2}+y_{S}^{2}\right)^{2}$

for $y_{I}$, which will generically have three real solutions. After obtaining $y_{I}$, we obtain two symmetric solutions for $x_{I}$ by

$x_{I}= \pm \sqrt{y^{2}\left(y_{S} / y_{I}-1\right)-a^{2}\left(y_{I} / y_{S}-1\right)}$.

Equation (A2) is cubic, and so we can generically get three real solutions. However, only negative $y_{I}$ solutions to equation (A2) are physical. To see this, after a little manipulating of the lens equation, we obtain $\lambda=-a^{2} y_{I} / y_{S}$, and since $\lambda$ is always positive, any images with $x_{I} \neq 0$ must have a $y_{I}$ coordinate opposite in sign to $y_{S}$, which means that we always get two such images, both with the same negative $y_{I}$-coordinate and with opposite $x_{I}$ coordinates.

This analysis allows us to understand why even very highly misaligned sources located along the $y_{S}$ axis can generate 3-images in the edge-on case (see the rightmost column of Figure 3). One of the three images is located at $x_{I}=0$ and $y_{I}>0$, while the other two are at located at $y_{I}<0$ and related to each other by reflection about the $y_{I}$ axis. At smaller $y_{S}$, the source creates four images, with the transition between the 3-image region and the 4-image region occurring at a critical source position of $y_{S, \mathrm{cr}}=a^{-1}$, below which equation (A1) has both positive and negative real solutions.

We now turn to diagnosing the situation for images along the $x_{S}$ axis. The general results are similar to those for images located along the $y_{S}$ axis. By beginning from the lens equation, we find images along that are located along the $x_{I}$ axis by solving

$\left(x_{I}-x_{S}\right)^{2}\left(x_{I}^{2}-a^{2}\right)=1$.

Note that this means that we only can form images along the $x_{I}$ axis if $x_{I}>a$. For images with nonzero $y_{I}$, we find $x_{I}$ by solving

$1-x_{S} / x_{I}=\left(x_{I} / x_{S}-1\right)^{2}\left(a^{2}-x_{S}^{2}\right)^{2}$.

Once we obtain $x_{I}$, we obtain two mirror symmetric solutions for $y_{I}$ given by

$y_{I}= \pm \sqrt{x_{I}^{2}\left(x_{S} / x_{I}-1\right)+a^{2}\left(x_{I} / x_{S}-1\right)}$.

\section{APPENDIX B: ANALYTIC CALCULATION OF THE MAGNIFICATION FOR THE RING/BELT+POINT SYSTEM}

The magnification for a ring/belt+point system at a given point in the source plane can be obtained analytically, which is very useful for performing numerical calculations and identifying the caustic set of a given lensing geometry. The magnification at a point $\boldsymbol{r}_{I}$ in the image plane is

$\mu\left(\boldsymbol{r}_{I}\right)=\frac{1}{\operatorname{det}(A)}$,

where the Jacobian of the lens mapping is given by

$A_{i j}=\delta_{i j}-\frac{\partial \alpha_{i}}{\partial x_{j}}$.

For the ring+point mass system, the Jacobian matrix is

$A=\left(\begin{array}{cc}1-g_{x x} & g_{x y} \\ g_{y x} & 1-g_{y y}\end{array}\right)$, where

$$
\begin{aligned}
& g_{x x}=\frac{1}{1+q} \frac{\left|\boldsymbol{r}_{I}\right|^{2}-2 x_{I}^{2}}{\left|\boldsymbol{r}_{I}\right|^{4}}+\frac{q}{1+q} \frac{\partial \alpha_{r, x}}{\partial x_{I}} \Theta\left(\frac{x_{I}^{2}}{a^{2}}+\frac{y_{I}^{2}}{b^{2}}-1\right), \\
& g_{x y}=g_{y x}=-\frac{1}{1+q} \frac{2 x_{I} y_{I}}{\left|\boldsymbol{r}_{I}\right|^{4}}+\frac{q}{1+q} \frac{\partial \alpha_{r, x}}{\partial y_{I}} \Theta\left(\frac{x_{I}^{2}}{a^{2}}+\frac{y_{I}^{2}}{b^{2}}-1\right), \\
& g_{y y}=\frac{1}{1+q} \frac{\left|\boldsymbol{r}_{I}\right|^{2}-2 y_{I}^{2}}{\left|\boldsymbol{r}_{I}\right|^{4}}+\frac{q}{1+q} \frac{\partial \alpha_{r, y}}{\partial y_{I}} \Theta\left(\frac{x_{I}^{2}}{a^{2}}+\frac{y_{I}^{2}}{b^{2}}-1\right) .
\end{aligned}
$$

The first terms in each equation are from the point mass, with the second terms coming from the ring's contribution. Here, $\Theta$ is the Heaviside step function and $\alpha_{r, x}\left(\alpha_{r, y}\right)$ denotes the $x$-component (y-component) of the deflection angle due to the ring. The partial derivatives of the deflection angle in equation (B4) can be computed analytically, yielding

$$
\begin{aligned}
& \frac{\partial \alpha_{r, x}}{\partial x_{I}}= \frac{p^{\prime 2}}{a^{\prime 5} b^{\prime}}\left(a^{\prime 2}-x_{I} \frac{\partial \lambda}{\partial x_{I}}\right)+\frac{2 p^{\prime 4} x_{I}}{a^{\prime 3} b^{\prime}}\left[\left(\frac{x_{I}^{2}}{a^{\prime 6}}+\frac{y_{I}^{2}}{b^{\prime}}\right) \frac{\partial \lambda}{\partial x_{I}}-\frac{x_{I}}{a^{\prime 4}}\right] \\
&-\frac{p^{\prime 2} x_{I}}{a^{\prime 4} b^{\prime 2}} \frac{\partial \lambda}{\partial x_{I}}\left(\frac{b^{\prime}}{2 a^{\prime}}+\frac{a^{\prime}}{2 b^{\prime}}\right), \\
& \frac{\partial \alpha_{r, y}}{\partial y_{I}}= \frac{p^{\prime 2}}{a^{\prime} b^{\prime 5}}\left(b^{\prime 2}-y_{I} \frac{\partial \lambda}{\partial y_{I}}\right)+\frac{2 p^{\prime 4} y_{I}}{a^{\prime} b^{\prime 3}}\left[\left(\frac{x_{I}^{2}}{a^{\prime 6}}+\frac{y_{I}^{2}}{b^{\prime 6}}\right) \frac{\partial \lambda}{\partial y_{I}}-\frac{y_{I}}{b^{\prime 4}}\right] \\
&-\frac{p^{\prime 2} y_{I}}{a^{\prime 2} b^{\prime 4}} \frac{\partial \lambda}{\partial y_{I}}\left(\frac{b^{\prime}}{2 a^{\prime}}+\frac{a^{\prime}}{2 b^{\prime}}\right), \\
& \frac{\partial \alpha_{r, x}=}{\partial y_{I}}=\frac{\partial \alpha_{y}}{\partial x_{I}}=-\frac{p^{\prime 2} x_{I}}{a^{\prime} 5 b^{\prime}} \frac{\partial \lambda}{\partial y_{I}}+\frac{2 p^{\prime 4} x_{I}}{a^{\prime 3} b^{\prime}}\left[\left(\frac{x_{I}^{2}}{a^{\prime 6}}+\frac{y_{I}^{2}}{b^{\prime 6}}\right) \frac{\partial \lambda}{\partial y_{I}}-\frac{y_{I}}{b^{\prime 4}}\right] \\
&-\frac{p^{\prime 2} x_{I}}{a^{\prime 4} b^{\prime 2}} \frac{\partial \lambda}{\partial y_{I}}\left(\frac{b^{\prime}}{2 a^{\prime}}+\frac{a^{\prime}}{2 b^{\prime}}\right),
\end{aligned}
$$

where the primed variables retain their meanings from section 2.2 [see equation (18)]. The partial derivatives of $\lambda$ are given by

$\frac{\partial \lambda}{\partial x_{I}}=\frac{2 p^{\prime 2} x_{I}}{a^{\prime 2}}$ and $\quad \frac{\partial \lambda}{\partial y_{I}}=\frac{2 p^{\prime 2} y_{I}}{b^{\prime 2}}$.

An analytic expression also exists for thick belts. Letting $a_{i}$ and $a_{o}$ be the inner and outer radii (respectively) of the belt, we compute the magnification as in equation (B4), but this time with the factors of $\Theta\left(x_{I}^{2} / a^{2}+y_{I}^{2} / b^{2}-1\right)$ replaced by $\Theta\left(x_{I}^{2} / a_{i}^{2}+y_{I}^{2} / b_{i}^{2}-1\right)$, and with the partial derivatives replaced with

$$
\begin{aligned}
& \frac{\partial \alpha_{b, x}}{\partial x_{I}}=2 a_{o} b_{o}\left(\frac{1}{a_{o}^{\prime}\left(a_{o}^{\prime}+b_{o}^{\prime}\right)}-\frac{\partial \lambda_{o}}{\partial x_{I}} \frac{x_{I}}{2 a_{o}^{\prime 3} b_{o}^{\prime}}\right) \\
& -2 a_{i} b_{i}\left(\frac{1}{a_{i}^{\prime}\left(a_{i}^{\prime}+b_{i}^{\prime}\right)}-\frac{\partial \lambda_{i}}{\partial x_{I}} \frac{x_{I}}{2 a_{i}^{\prime 3} b_{i}^{\prime}}\right), \\
& \frac{\partial \alpha_{b, y}}{\partial y_{I}}=2 a_{o} b_{o}\left(\frac{1}{b_{o}^{\prime}\left(a_{o}^{\prime}+b_{o}^{\prime}\right)}-\frac{\partial \lambda_{o}}{\partial y_{I}} \frac{y_{I}}{2 a_{o}^{\prime} b_{o}^{\prime 3}}\right) \\
& -2 a_{i} b_{i}\left(\frac{1}{b_{i}^{\prime}\left(a_{i}^{\prime}+b_{i}^{\prime}\right)}-\frac{\partial \lambda_{i}}{\partial y_{I}} \frac{y_{I}}{2 a_{i}^{\prime} b_{i}^{\prime 3}}\right), \\
& \frac{\partial \alpha_{b, x}}{\partial y_{I}}=\frac{\partial \alpha_{y}}{\partial x_{I}}=2 a_{o} b_{o}\left(\frac{\partial \lambda_{o}}{\partial y_{I}} \frac{-x_{I}}{2 a_{o}^{\prime 3} b_{o}^{\prime}}\right)-2 a_{i} b_{i}\left(\frac{\partial \lambda_{i}}{\partial y_{I}} \frac{-x_{I}}{2 a_{i}^{\prime 3} b_{i}^{\prime}}\right) \text {. }
\end{aligned}
$$

The primed semi-axes are defined through

$a_{i}^{\prime 2}=a_{i}^{2}+\lambda_{i}, \quad b_{i}^{\prime 2}=b_{i}^{2}+\lambda_{i}, \quad a_{o}^{\prime 2}=a_{o}^{2}+\lambda_{o}, \quad b_{o}^{\prime 2}=b_{o}^{2}+\lambda_{o}$,

and

$p_{i}^{\prime}=\left(x_{I}^{2} / a_{i}^{\prime 4}+y_{I}^{2} / b_{i}^{\prime 4}\right)^{-1 / 2}, \quad p_{o}^{\prime}=\left(x_{I}^{2} / a_{o}^{\prime 4}+y_{I}^{2} / b_{o}^{\prime 4}\right)^{-1 / 2}$

are the perpendicular distances from the center of the inner and outer confocal ellipses to the tangent lines at $\boldsymbol{r}_{I}$, respectively. 
As in the thin ring case, $\lambda_{i}\left(\lambda_{o}\right)$ is found by solving equation (18) and using $a=a_{i}$ and $b=b_{i}\left(a=a_{o}\right.$ and $\left.b=b_{o}\right)$. The partial derivatives of $\lambda_{i}$ are

$\frac{\partial \lambda_{i}}{\partial x_{I}}=\frac{2 p_{i}^{\prime 2} x_{I}}{a_{i}^{\prime 2}} \quad$ and $\quad \frac{\partial \lambda_{i}}{\partial y_{I}}=\frac{2 p_{i}^{\prime 2} y_{I}}{b_{i}^{\prime 2}}$

while those of $\lambda_{o}$ are

$\frac{\partial \lambda_{o}}{\partial x_{I}}=\frac{2 p_{o}^{\prime 2} x_{I}}{a_{o}^{2}} \Theta\left(\frac{x_{I}^{2}}{a_{o}^{2}}+\frac{y_{I}^{2}}{b_{o}^{2}}-1\right)$ and $\frac{\partial \lambda_{o}}{\partial y_{I}}=\frac{2 p_{o}^{\prime 2} y_{I}}{b_{o}^{\prime 2}} \Theta\left(\frac{x_{I}^{2}}{a_{o}^{2}}+\frac{y_{I}^{2}}{b_{o}^{2}}-1\right)$.

We note that for a ring or disc with uniform density Blandford et al. (1991) derive the gradient of the deflection angle with an alternative method.

\section{APPENDIX C: EXPLICIT EXPRESSION FOR THE}

\section{PSEUDO-CAUSTICS}

We now present an explicit expression for the pseudo-caustics of a belt+point mass system at arbitrary inclination by mapping the inner and outer edge of the belt to the source plane. There are two sets of pseudo-caustics, which we will label as $\mathcal{P} \mathcal{S}_{1}$ and $\mathcal{P} \mathcal{S}_{2}$. They are parametrized by an angle $\phi \in[0,2 \pi)$ and given by

$\mathcal{P} \mathcal{S}_{1}(\phi)=\left(a_{i} \cos \phi \hat{\boldsymbol{x}}_{S}+b_{i} \sin \phi \hat{\mathbf{y}}_{S}\right)\left(1-\frac{1}{1+q} \frac{1}{a_{i}^{2} \cos ^{2} \phi+b_{i}^{2} \sin ^{2} \phi}\right)$

and

$$
\begin{aligned}
& \mathcal{P S}_{2}(\phi)=a_{o} \cos \phi \hat{\boldsymbol{x}}_{S}\left[1-\frac{1}{(1+q)\left(a_{o}^{2} \cos ^{2} \phi+b_{o}^{2} \sin ^{2} \phi\right)}\right. \\
& \left.-\frac{q}{(1+q)\left(a_{o} b_{o}-a_{i} b_{i}\right)}\left(\frac{2 b_{o}}{a_{o}+b_{o}}-\frac{2 a_{i} b_{i}}{\left(a_{i}^{\prime}(\phi)+b_{i}^{\prime}(\phi)\right) a_{i}^{\prime}(\phi)}\right)\right] \\
& +b_{o} \sin \phi \hat{\boldsymbol{y}}_{S}\left[1-\frac{1}{(1+q)\left(a_{o}^{2} \cos ^{2} \phi+b_{o}^{2} \sin ^{2} \phi\right)}\right. \\
& \left.-\frac{q}{(1+q)\left(a_{o} b_{o}-a_{i} b_{i}\right)}\left(\frac{2 a_{o}}{a_{o}+b_{o}}-\frac{2 a_{i} b_{i}}{\left(a_{i}^{\prime}(\phi)+b_{i}^{\prime}(\phi)\right) b_{i}^{\prime}(\phi)}\right)\right],
\end{aligned}
$$

where

$a_{i}^{\prime}(\phi)=\sqrt{a_{i}^{2}+\lambda_{i}(\phi)}$ and $b_{i}^{\prime}(\phi)=\sqrt{b_{i}^{2}+\lambda_{i}(\phi)}$

with

$$
\begin{aligned}
\lambda_{i}(\phi) & =\frac{a_{o}^{2} \cos ^{2} \phi+b_{o}^{2} \sin ^{2} \phi-a_{i}^{2}-b_{i}^{2}}{2} \\
& +\left[\frac{1}{4}\left(a_{o}^{2} \cos ^{2} \phi+b_{o}^{2} \sin ^{2} \phi-a_{i}^{2}-b_{i}^{2}\right)^{2}\right. \\
& \left.-a_{i}^{2} b_{i}^{2}+b_{i}^{2} a_{o}^{2} \cos ^{2} \phi+a_{i}^{2} b_{o}^{2} \sin ^{2} \phi\right]^{1 / 2} .
\end{aligned}
$$

